\title{
Contention-Detectable Mechanism for Receiver-Initiated MAC
}

\author{
DAIBO LIU, Hunan University, China \\ ZHICHAO CAO, Tsinghua University, China \\ MINGYAN LIU, University of Michigan, USA \\ MENGSHU HOU, University of Electronic Science and Technology of China, China \\ HONGBO JINAG, Hunan University, China
}

\begin{abstract}
The energy efficiency and delivery robustness are two critical issues for low duty-cycled wireless sensor networks. The asynchronous receiver-initiated duty-cycling media access control (MAC) protocols have shown their effectiveness through various studies. In receiver-initiated MACs, packet transmission is triggered by the probe of receiver. However, it suffers from the performance degradation incurred by packet collision, especially under bursty traffic. Several protocols have been proposed to address this problem, but their performance is restricted by the unnecessary backoff time and long negotiation process. In this article, we present $\mathrm{CD}-\mathrm{MAC}$, an energy-efficient and robust contention-detectable mechanism for addressing the collision-catching problem in receiver-initiated MACs. By exploring the temporal diversity of the acknowledgments, a receiver recognizes the potential senders and subsequently polls individual senders one by one. On that basis, CD-MAC can successfully avoid packet collision even though multiple senders have data packets to transmit to the same receiver. We implement CD-MAC in TinyOS and evaluate its performance on an indoor testbed with single-hop and multi-hop network scenarios. The results show that CD-MAC can significantly improve throughput by 1.72 times compared with the state-of-the-art receiver-initiated MAC protocol under bursty traffic loads. The results also demonstrate that CD-MAC can effectively mitigate the influence of hidden terminal problem and adapt to network dynamics well.
\end{abstract}

CCS Concepts: • Networks $\rightarrow$ Link-layer protocols; $\bullet$ Computer systems organization $\rightarrow$ Sensor networks;

Additional Key Words and Phrases: Wireless sensor networks, receiver-initiated MAC, contention avoidance, temporal diversity

This work was partially supported by the National Key Research and Development Program of China (2018YFC0831800), NSFC 61772184, 61732017, 61502162, 61572219, 61702175, NSF under grant ECCS 1446521, and the Fundamental Research Funds for the Central Universities.

Authors' addresses: D. Liu (corresponding author), Hunan University, Changsha, Hunan Province, China; email: dbliu@hnu.edu.cn; Z. Cao, Tsinghua University, Beijing, China; email: caozc@mail.tsinghua.edu.cn; M. Liu, University of Michigan, Ann Arbor, Michigan, USA; email: mingyan@umich.edu; M. Hou, University of Electronic Science and Technology of China, Chengdu, Sichuan Province, China; email: mshou@uestc.edu.cn; H. Jinag, Hunan University, Changsha, Hunan Province, China; email: hongbojiang@hnu.edu.cn.

Permission to make digital or hard copies of all or part of this work for personal or classroom use is granted without fee provided that copies are not made or distributed for profit or commercial advantage and that copies bear this notice and the full citation on the first page. Copyrights for components of this work owned by others than ACM must be honored. Abstracting with credit is permitted. To copy otherwise, or republish, to post on servers or to redistribute to lists, requires prior specific permission and/or a fee. Request permissions from permissions@acm.org.

(C) 2019 Association for Computing Machinery.

1539-9087/2019/06-ART31 \$15.00

https://doi.org/10.1145/3317683

ACM Transactions on Embedded Computing Systems, Vol. 18, No. 4, Article 31. Publication date: June 2019. 


\section{ACM Reference format:}

Daibo Liu, Zhichao Cao, Mingyan Liu, Mengshu Hou, and Hongbo Jinag. 2019. Contention-Detectable Mechanism for Receiver-Initiated MAC. ACM Trans. Embed. Comput. Syst. 18, 4, Article 31 (June 2019), 27 pages. https://doi.org/10.1145/3317683

\section{INTRODUCTION}

Wireless sensor networks (WSNs) are replacing the traditional wired industrial communication systems since the industrial WSNs (IWSNs) offer several advantages including easy and fast installation and low-cost maintenance [11,27].

IWSN/WSN applications [21, 28, 40] are widely used in many different scenarios [22, 23, 40]. Due to the constraint of energy resource, the radio of sensor motes usually works in the low-duty cycle mode [30] to extend network lifetime. A widely adopted low-duty cycle media access control (MAC) is asynchronous receiver-initiated, such as the pioneered RI-MAC [35] and the state-of-theart A-MAC [8]. In A-MAC, for example, nodes periodically wake up and send a data solicitation (called probe in this article) to notice potential senders [25]. When a packet is pending, a sender waits the intended receiver's probe. Then it sends the packet after successfully decoding the probe. Many works [8, 25, 26, 35] have reported the energy efficiency and versatility of this scheme.

One critical issue [26] of receiver-initiated MAC is that the opportunity of data reception is decreased, because the radio is turned off most of the time. Senders having data packet to transmit should keep silent until the corresponding receivers wake up, and then immediately transmit their data packets. As a result, the probability of packet collisions increases [38]. This problem will be further aggravated when the traffic load is high or an unexpected surge of traffic occurs, because there is likely to be multiple senders having data packets to transmit to the same receiver. We refer to this situation as a collision-catching problem. Consider an event-driven network [2, 13, 17], nodes keep silence until an event occurs. Upon detecting the event, several nodes simultaneously report it, leading to sudden bursts of traffic [17, 26]. Beyond that, network image updates $[14,19]$ and periodic data collection with high sampling ratio [33] also can generate a lot of traffic within a short time. Thus, it is of great importance to design an efficient contention-resolution mechanism.

For the receiver-initiated duty-cycling MAC, it allows a receiver to coordinate the potential senders by either dynamically adjusting the backoff period $[8,35]$ or involving extra negotiation process $[18,26]$. Although these existing methods alleviate the contention problem in some degree, some innate limitations still hamper their performance. First, enlarging the backoff period to avoid collision will reduce energy efficiency and throughput. Second, the extra negotiation process will increase the energy baseline. Finally, some requirements of the auxiliary information (synchronization, signal strength, packet length, etc.) [6] might limit the scalability.

On the other hand, the way that a receiver coordinates all senders based on traditional TDMA reservation approaches [10], such as DW-MAC [34], AS-MAC [41], BN-MAC [32], also holds several limitations. A notable drawback [31] is that due to the fixed slotted schedule, TDMA-based reservation approaches are hard to achieve adaptive duty-cycle for diverse traffic patterns. Moreover, the reliability depends on the synchronization accuracy between receiver and senders, consuming extra maintenance overhead.

The real difficulty for solving the innate collision-catching problem in receiver-initiated MACs is the incompatible contradiction between distributed working manner of sensor nodes and the needing of the information of potential senders for making the optimal transmission decision. By far, although various resolutions have been proposed to address the channel contention problem, there are still no effective resolution for receiver-initiated MACs. 
To address the channel contention problem, we present CD-MAC, a Contention-Detectable mechanism for solving collision-catching problem of receiver-initiated MACs. CD-MAC integrates a lightweight contention resolution mechanism into the existing framework of receiver-initiated MAC. The basic idea is that the receiver utilizes the temporal diversity [20] of multiple quickly replied acknowledgments (ACK) to recognize the potential senders, then it will poll the senders' data packets one by one. Since the period of one ACK transmission is very short ( $352 \mu$ s for CC 2420 [16]), the overhead of coordination process is low. CD-MAC also does not need any auxiliary control overhead in distinguishing potential senders. Furthermore, CD-MAC uses double-modulo operation to guarantee that potential contending senders will select a different time slot to reply ACKs in a distributed manner. According to the contention detectable mechanism of CD-MAC, it is feasible to guarantee energy efficiency and network reliability for various network applications built upon receiver-initiated link layer.

The main contributions of this work are as follows:

(1) We develop a practical and novel contention resolution mechanism to solve the innate collision-catching problem of receiver-initiated MACs by utilizing the temporal diversity of ACKs replied by multiple senders.

(2) We propose CD-MAC to improve the performance of asynchronous receiver-initiated duty cycling protocols for various traffic patterns (periodic, bursty, and mobility).

(3) We have implemented CD-MAC in TinyOS-2.1.1 [39] and evaluated its performance on an indoor testbed. The results demonstrate that CD-MAC can improve throughput by up to $172 \%$ compared with the state-of-the-art receiver-initiated MAC protocols under bursty traffic loads with low control overhead. The results also demonstrate that CD-MAC can effectively mitigate the influence of hidden terminal problems and adapt to network dynamics well.

The remainder of this article is organized as follows: We first discuss the related work in the next section. In Section 3, we give the detailed design of CD-MAC. Then, we present its implementation and evaluation in Sections 4 and 5, respectively. We discuss the limitations of CD-MAC in Section 6. Finally, we conclude this article in Section 7.

\section{RELATED WORK}

Channel contention resolution has been extensively studied and been tackled from various aspects in research literatures. Researchers have proposed numerous protocols and mechanisms across different network layers. Here we have a brief discussion on the existing proposals in three aspects respectively.

Carrier sensing and backoff is a basic channel contention mechanism. Due to its simple algorithm, good robustness, and the high merit of reliability, it has been widely applied by current MAC protocols, such as RI-MAC [35], X-MAC [3], BoX-MAC [24], A-MAC [8], EM-MAC [36], and PW-MAC [37]. They exploit basic carrier sensing to guarantee that, in a specific time slot, there should be no more than one sender transmitting, while the other senders need to perform random backoff to avoid collision. In networks with low traffic load, MACs adopting carrier sensing and backoff for channel contention can achieve high efficient and reliable data forwarding. However, with the increasing of traffic load, the advantages of carrier sensing and backoff mechanism are gradually reducing, and the disadvantage of carrier sensing and backoff mechanism, i.e., ineffective backoff for channel contention and weak ability in addressing data collision, could significantly degrade the performance of data forwarding. The obvious advantages and limitations of the basic carrier sensing and backoff mechanism make it widely applied by practically deployed low-power wireless networks and strongly limits the application scenarios. 
Another part of MAC protocols schedule senders' transmissions to eliminate collisions. TCF [29] dynamically allocates channel resource to get rid of contention overhead of CSMA. Z-MAC [15] proposes a hybrid MAC protocol combining CSMA and TDMA to be adaptive to dynamic traffic loads, however, at the cost of remarkable overhead incurred by synchronization and coordination. BN-MAC [32] and O-MAC [1] support the hybrid topology that combines the features of both TDMA and CSMA. They adopt TDMA feature to schedule neighbors with unique transmission slot to address channel contention problem, but in multi-hop sensor networks, their performance could be seriously degraded due to long-delayed transmission and low energy efficiency. DWMAC [34] and AS-MAC [41] allow sensor nodes to wake up on demand during the sleep period of a duty cycle to transmit or receive packets with a mapping function to prevent data collision, but they require synchronized duty cycle of all network nodes, which can bring about significant synchronization overhead for low power sensor networks. All those resolutions thus have limited applicability in large-scale sensor networks.

Some other channel contention resolutions $[18,26]$ are proposed to embed to existing MAC protocols by using deliberate contention. Although these approaches alleviate the contention problem in some degree, however, the using of the enlarging of backoff period for collision avoidance could reduce energy efficiency and throughput. In addition, the extra negotiation process will also increase the energy baseline of the whole networks. For this kind of contention resolutions, the requirements of the auxiliary information (e.g., synchronization, signal strength, packet length) might limit the scalability.

Unlike the existing approaches, CD-MAC utilizes the temporal diversity of quickly replied ACKs to differentiate potential senders. By using the proposed ACK slot-selecting approach, contending senders will select different slots to quickly reply ACKs so as to announce their intention to send data to their common receiver. Then, the receiver could poll the senders one by one for their packets. Compared with all of the above-mentioned approaches, CD-MAC can resolve the contention problem with outstanding scalability; moreover, the energy/control overhead of CD-MAC is negligible. Based on this mechanism, CD-MAC can effectively and efficiently eliminate the contention between potential senders and avoid data collision with very low overhead.

\section{DESIGN OF CD-MAC}

\subsection{Design Overview}

Under a receiver-initiated MAC design framework, the basic operation of CD-MAC is given in this section, as described below. A sender listens for transmission solicitation from the intended receiver. The receiver, upon waking up, immediately sends a probe if the channel is sensed to be idle, notifying all potential senders within its range that it is ready to receive packets, as well as initiating a reservation frame (referred to as an RSVP frame) containing a certain number of slots immediately following the probe. A sender, upon receiving the probe, acknowledges it using an ACK sent within a selected slot in the reservation frame, thereby announcing its intention of sending data to the receiver. At the completion of the reservation frame, the receiver obtains the information on the quantity of nodes which are ready to send data to it and the difference between them by exploring the temporal diversity, with the possibility of ACKs lost due to corruption or collision when multiple ACKs happen to be sent within the same slot. The receiver then polls each of the pronounced senders, one at a time, for data transmission. The amount of data sent by each sender upon being polled is adjustable and can be catered to the need of practical applications.

This operation is depicted in Figure 1. $S_{i}(1 \leq i \leq 5)$ holds data and waits for the intended receiver $R$ to wake up. When $R$ wakes up, it sends a Sender Probe (referred to as an $S$-probe or $C$ in all figures) packet to notify senders of its readiness to receive, and to indicate the start of a reservation 


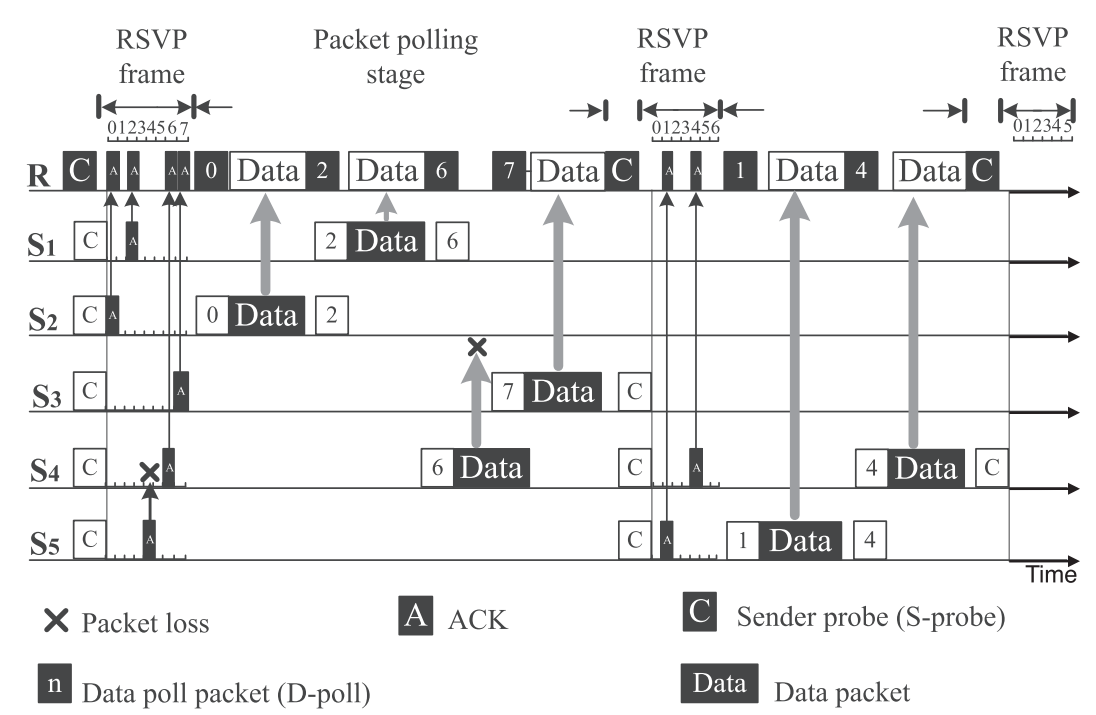

Fig. 1. Overview of CD-MAC, which consists of reservation frame and packet polling stage. Among the duration of reservation frame, senders reply ACKs by selecting different slots to confirm the success reception of S-probe $(C)$. During the packet polling stage, the receiver $R$ polls senders by D-poll (dark square with a number) in orderly manner according to the acknowledged slot sequence number. CD-MAC also uses D-poll to acknowledge previous data packet. If a D-poll (attaching with an appointed slot number 6) doesn't solicit a data, $R$ continues to solicit another sender's data.

frame which consists of $n$ time slots. Parameters attached in the S-probe are two integers, $n$ and $m$. $m$ is an auxiliary parameter used by each sender to calculate its slot number (the position in the reservation frame) in which to send its ACK (details of the computation is given in the following section). Both $n$ and $m$ can vary from one solicitation to another. During the reservation frame, an ACK could be lost due to corruption (e.g., the ACK sent by $S_{5}$ as illustrated in Figure 1) or collision when multiple senders happen to select the same slot number to reply ACKs. In such cases, the receiver could not perceive the intention of these senders whose ACKs were lost. Those whose ACKs have been successfully received by the receiver will be referred to as announced senders.

Following the reservation frame, the receiver starts the packet polling stage, in which it polls the announced senders one by one. Each polling starts with a Data-poll (referred to as D-poll or P in all figures) packet, which also serves as an ACK to acknowledge the previously received data packet, if there is one. This process is repeated until the receiver has polled all the announced senders. Within each poll, the polled sender can choose to send one or several of its data packets, i.e., each poll may occupy different amount of time. At the conclusion of the data polling stage, the receiver will transmit a S-probe to acknowledge the last sender, and may conduct another solicitation once a sender announces its intention of sending data. Otherwise, the receiver goes to sleep mode soon.

Note that CD-MAC assigns a different number of slots to successive RSVP phases. Hence a sender whose ACK or data was corrupted at receiver side due to collision will again announce its intention of sending data to the receiver by selecting a different slot. Then, the conflicting senders in previous RSVP phase are likely to select different slots in the next RSVP phase so as to avoid collision again. The adoption of variable $n$ aims to make the tradeoff between the adaptivity of variant network traffic, network dynamics, and energy efficiency. Because in silent phase network traffic is low, a small number of slots are enough to differentiate potential senders. However, in a network with sudden surge of traffic load, an increasing number of slots are needed to differentiate 
the potentially increased senders. Considering this situation, using RSVP with variable slot number is much more adaptive to network dynamics than the method with fixed slot [5]. The selection of parameters $n$ and $m$, as well as other design details are given below.

\subsection{Reservation Frame Size and Slot Computation}

One of the most critical design parameters of our scheme is the size of a reservation frame $(n)$ and the procedure a sender uses to determine its slot position in the RSVP frame using the two integers $n$ and $m$. The objective is to do it in such a way so that the senders will select slot numbers that tend to spread out across the frame so as to minimize the probability of collision.

We now detail the procedure that a sender $S$ uses to determine its slot number. The input to this process consists of $n, m$, as well as the sender's network-wise identification (ID) number denoted by $s$, and the output is the slot number denoted by $\pi(s, m, n)$. The procedure takes two steps: $S$ first maps its ID $s$ into an integer within $[1, m]$, then further into the range $[1, n]$, both using a modulo operation:

$$
\pi(s, m, n)=\operatorname{Mod}(\operatorname{Mod}(s, m), n) .
$$

Note that $s$ is the identity of a specific neighbor node, but its value can range from 1 to a very large number. The rationale behind this double-modulo process is to provide one more degree of freedom so we could achieve a better tradeoff between having low delay (which requires a relatively small value of $n$ ) and low collision ratio over slot selection (which requires a sufficiently large value of $n$ ). To make the double-module process work well, $m$ must be larger than $n$.

3.2.1 Optimal Choice of $m$. Specifically, we let a receiver first fix the value for $n$, chosen from a pre-set range $\left[n_{\min }, n_{\max }\right]$. The dynamic selection of $n$ is detailed shortly. The selection of the value for $m$ is then determined by the following minimization problem:

$$
\min _{m} \sum \sum_{i \neq j} I_{\left\{\pi\left(s_{i}, m, n\right)=\pi\left(s_{j}, m, n\right)\right\}}
$$

where $s_{i}, s_{j}$ denote a pair of nodes in the network, and the indicator function $I_{A}=1$ if $A$ is true and 0 otherwise. The above objective function seeks to minimize the total pair-wise collisions in slot selection. The optimal $m$ values can then be computed for each selection of $n$ values. CDMAC assumes the vast majority of children nodes' IDs are known. Hence, as a receiver, it uses the IDs of all the children nodes to select the optimal value of $m$ for a specific $n$. This assumption is reasonable because a node can easily collect the topological relation by overhearing network beacons in the initial routing constructing and the later network maintenance. The computation is triggered when network topology has been constructed. Then, CD-MAC gets several optimal parameter pairs, and selects one of them before sending each S-probe. The computation will also be triggered again when a new arrival child node is found and the slot collision ratio between potential senders exceeds a pre-defined threshold value. Note that the computation complexity is $O(\overline{m n c})$, where $\bar{m}$ is the space size of $m, \bar{n}$ is the space size of $n$, and $\bar{c}$ is the amount of child nodes. The cost of computation is usually very small because of limited $\bar{n}$ and $\bar{c}$. For efficiency, we set the range of $m$ from 0 to 1000 in our implementation. More precisely, $m$ should be larger than $n$ to make the double-module process (Equation (1)) effective.

To demonstrate the feasibility and effectiveness of the double-module process, we conduct a simulation on a normal computer. Considering that CD-MAC is a general receiver-initiated MAC applicable to various application scenarios, we don't make assumptions on the distribution model of network nodes. In this simulation, a node's neighbors are randomly assigned. The number of neighbors can be $5,10,15$, and 20 in the simulations. As receiver, once the neighbors have been assigned, it first computes the optimal parameter value of $m$ corresponding to different number of 


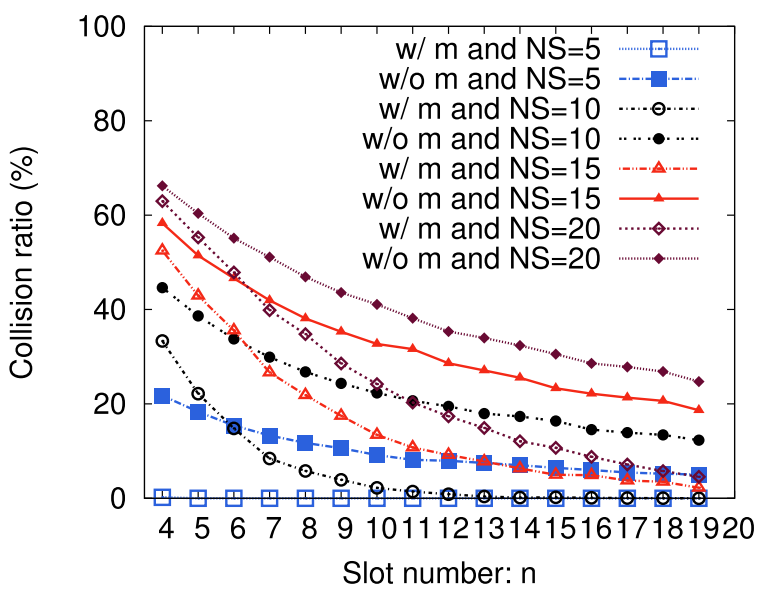

Fig. 2. ACK collision ratio as a function of the reservation frame size $n$, with and without using $m$. Node IDs are from $[0,1,000]$.

slots $(n)$. Then, by changing the number of slots and randomly selecting a portion of neighbors to generate data packets and transmit to the same receiver, when these senders receive a S-probe that carries a pair of of $n$ and $m$, they respectively compute ACK slots according to the above-mentioned double-module process. Then, we can compute the slot conflict probability. The effectiveness of the two-pass modulo operation is demonstrated in Figure 2, where we plot the simulated ACK collision ratio after the node IDs are mapped into multiple slots (ranging from 4 to 20) within the reservation frame, as a function of the frame size $n$. Here a node ID $s \in[0,1000] ; m$ is ranging from 0 to 1,000; up to NS (NS denotes the number of neighbor nodes) node IDs are randomly selected from this set to represent the number of neighbor nodes. We then compare the collision ratio between using a two-pass process (i.e., collisions based on mapping the node IDs using $\pi(s, m, n)$ with an optimal choice of $m$; denoted as $w / m$ in the figure) and not using a two-pass process (i.e., collisions based on mapping the node IDs using $\pi(s, n, n)$; denoted as $w / o m$ in the figure). As shown by the simulation results, there is a dramatic reduction in collision ratio. In particular, with a neighborhood size limited to 5 , this two-pass modulo process practically reduces the ACK collision to zero.

3.2.2 Adaptive Selection of $n$. The selection of $n$ consists of two stages. One is to select an initial $n$ for the RSVP frame after a node's wakeup, and the other is to select a different $n$ to differentiate the potential senders which selected the same slot in the previous packet polling round.

For the former, the purpose is to increase $n$ if a receiver perceives high volume of traffic or large number of senders, and decrease $n$ otherwise. Consider that the traffic load for a specific receiver has time pertinence. Because the wakeup interval is very small, the traffic load of the next time to wake up is likely to be close to that of the previous wakeup. Hence, we use the moving average to calculate the expected slot number $n_{\text {exp }}$ for the next time to wake up. $n_{\text {old }}$ is the accumulated value of slot number and $n_{\text {new }}$ is the number of senders that had data to transmit when the receiver previously woke up. We update the accumulated value by

$$
n_{\text {exp }}=\alpha * n_{\text {old }}+(1-\alpha) * n_{\text {new }},
$$

where $\alpha$ is smoothing factor. For the latter, after a node's wakeup, it may conduct several RSVP frames and packet polling stages, as shown in Figure 1. All the potential senders will acknowledge the receiver by selecting different slots in the first RSVP frame, and (almost) most of the senders 


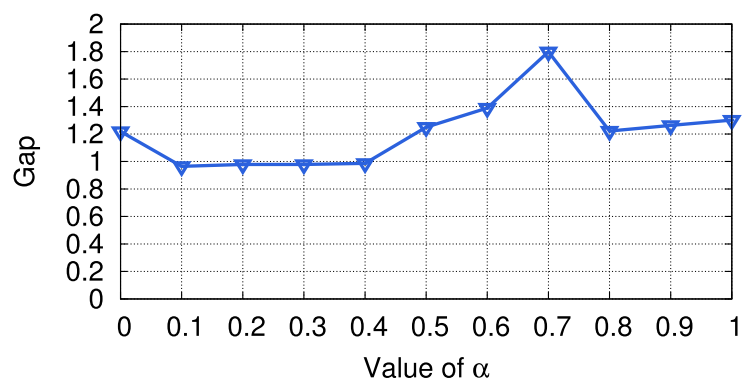

Fig. 3. The gap between the number of expected senders and the number of factual senders.

could successfully make reservation for their data transmission. Hence, the receiver could poll these senders that have made an appointment in the first packet polling stage. For the next RSVP frame, the purpose is to further differentiate the possible senders that selected the same slot to reply ACKs in the previous RSVP frame and solicit new senders. For simplicity, CD-MAC updates $n$ by alternatively decreasing 1 or increasing 1 .

To get a rational value of $\alpha$, we conduct experiment in an indoor testbed with 30 Telosb nodes. By selecting 6 relays in the data collection network, we trace the amount of senders in each wakeup interval of these relay nodes. The number of children nodes ranges between 4 and 13, and this experiment lasts an entire day. By setting different value to $\alpha$, we get the average gap between the actual amount of senders and the expected amount of senders $\left(n_{\exp }\right)$ as shown in Figure 3. To achieve a better tradeoff between having low gap and high adaptation to dynamics, 0.4 is a rational value for $\alpha$. By setting $n$ to be $n_{\exp }$ plus 2, the vast majority of packets can be polled during the first round. Note that the optimal value of $\alpha$ changes with data transmission ratio, a robust and efficient method should dynamically adjust the parameter. Some machine learning methods [12] could be used to adjust $\alpha$. However, for simplicity, we use a fixed experimental value in our implementation.

3.2.3 Tradeoff between Collision Ratio and Computation Overhead. Note that the arrival of new children nodes could increase the collision ratio of potential senders' slot selection. Beyond that, if CD-MAC immediately triggers the computation of the optimal $n$ and $m$, it could bring about heavy computation overhead. Considering both slot selection conflict and computation overhead, CD-MAC makes a tradeoff between them by setting a threshold of collision ratio. By detecting a new sender, based on the previously computed optimal parameters, if the expected collision ratio (ECR) exceeds the threshold, CD-MAC should trigger the recalculation of the optimal $n$ and the corresponding $m$.

With the same setup of simulation in Section 3.2.1, we further simulate this case by adding a new child node assigned with random and unique ID to an existing network. As the parent node of the new arriving node, it first computes the ECR when all potential senders (including the new arrival) simultaneously transmit data packets, and then it further computes the actual collision ratio (ACR) when different proportions of senders (as shown in the first column in Table 1) transmit data packets during the same polling stage by using the optimal $n$ and $m$. By roughly dividing the ECR space into four subsets ([0,20\%], [20\%, 30\%], [30\%, 40\%], and [40\%, 50\%]), which accounts for more than $99 \%$ of the ECRs in the simulated networks. We record the ACRs of each node under the condition that a given proportion of senders transmit data packets during the same polling stage. Then, we calculate the mean ACR and maximum ACR under different conditions, and list the results in Table 1 . To guarantee a relatively low actual collision ratio (ACR $<20 \%$ ) between potential senders, CD-MAC roughly sets the collision ratio threshold to $30 \%$, which can suppress almost $89 \%$ computations for reselecting the optimal parameter. In the rest of the article, 
Table 1. Actual Slot Collision Ratio of Potential Senders under the Conditions of Different Expected Collision Ratio and Different Proportions of Senders

\begin{tabular}{|c|c|c|c|c|c|c|c|c|}
\hline \multirow{2}{*}{$\begin{array}{c}\text { Ratio of concurrent senders } \\
\text { to all potential senders }\end{array}$} & \multicolumn{2}{|c|}{$E C R \in[0 \%, 20 \%]$} & \multicolumn{2}{|c|}{$E C R \in[20 \%, 30 \%]$} & \multicolumn{2}{|c|}{$E C R \in[30 \%, 40 \%]$} & \multicolumn{2}{|c|}{$E C R \in[40 \%, 50 \%]$} \\
\hline & mean & $\max$ & mean & $\max$ & mean & $\max$ & mean & $\max$ \\
\hline$[0,25 \%]$ & $0.05 \%$ & $5.73 \%$ & $3.62 \%$ & $7.27 \%$ & $4.83 \%$ & $9.85 \%$ & $6.35 \%$ & $12.40 \%$ \\
\hline$[25 \%, 50 \%]$ & $2.88 \%$ & $10.19 \%$ & $7.34 \%$ & $13.31 \%$ & $10.29 \%$ & $17.24 \%$ & $15.74 \%$ & $23.52 \%$ \\
\hline$[50 \%, 75 \%]$ & $4.81 \%$ & $14.93 \%$ & $12.57 \%$ & $19.57 \%$ & $17.28 \%$ & $24.89 \%$ & $24.54 \%$ & $35.26 \%$ \\
\hline$[75 \%, 99 \%]$ & $7.87 \%$ & $19.84 \%$ & $19.83 \%$ & $27.83 \%$ & $25.80 \%$ & $35.02 \%$ & $32.63 \%$ & $46.60 \%$ \\
\hline Percentage of different ECR & \multicolumn{2}{|c|}{$73 \%$} & \multicolumn{2}{|c|}{$16 \%$} & \multicolumn{2}{|c|}{$7 \%$} & \multicolumn{2}{|c|}{$3 \%$} \\
\hline
\end{tabular}

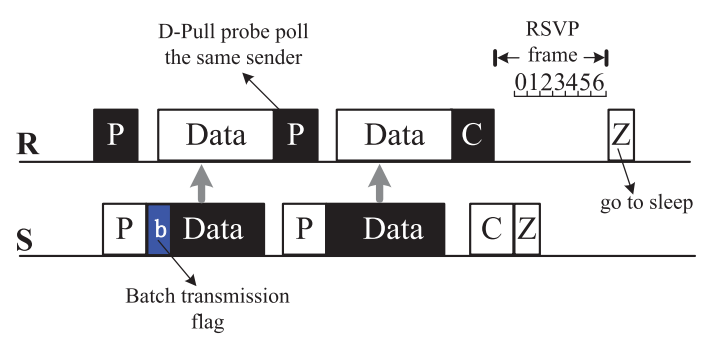

Fig. 4. Batch transmission. To successively transmit the holding data packets, $S$ sets the frame pending flag (b) in FCF of packet header. By receiving a flagged data packet, $R$ will poll $S$ again. $Z$ denotes a node turns into sleeping mode.

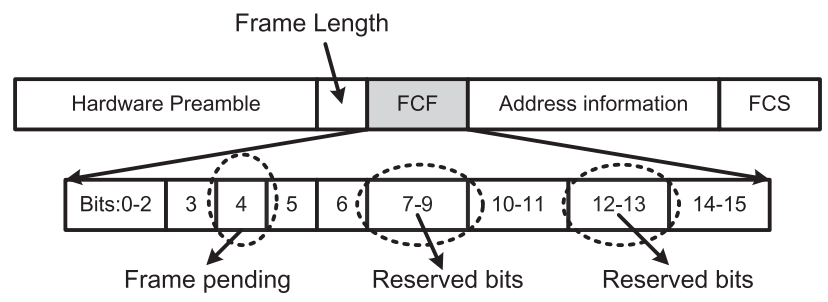

Fig. 5. The 4th bit of FCF is used to achieve batch transmission, the 5 reserved bits are used to attach the slot sequence number.

all evaluations are conducted by using the same threshold. Note that the selecting of the optimal threshold is complicated. We leave this problem to future work. Besides, as practical network deployers, they can also measure the optimal threshold for CD-MAC.

\subsection{Other Design Considerations}

3.3.1 Batch Transmission. If a sender has multiple packets to transmit, CD-MAC can support batch operation as follows: Upon being polled by its receiver, the sender sends the first packet with the batch transmission bit (flag $b$ in Figure 4) which is marked as frame pending of the MAC layer header in Figure 5 to inform the receiver that there is at least one other data packet. Upon receiving the packet, the receiver will then poll the same sender again with another D-poll packet.

3.3.2 Handling Packet Loss. A polled packet may not be successfully received because of data corruption, the moving of senders, or the mismatching of the ACK slot between the sender's selection and the receiver's inference. To address this issue, the receiver sets a timeout timer for each 


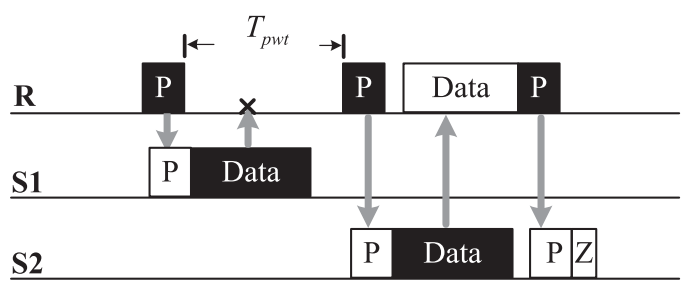

Fig. 6. Handling packet loss. If the data packet from $S_{1}$ is lost and the packet waiting time of $R$ exceeds $T_{p w t}$, $R$ moves on to $S_{2}$.

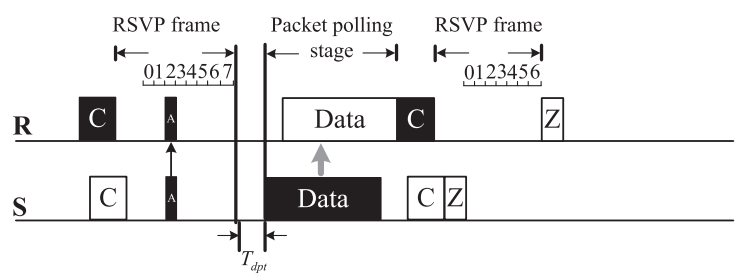

Fig. 7. Against a singular announced sender, $R$ does not poll $S$ by receiving only one ACK. Without listening, a D-poll from $R$ within $T_{d p t}, S$ immediately transmits its packet to $R$.

D-poll packet. When the timer expired and no data packet was received after the D-poll packet, the receiver transmits another D-poll packet to solicit the next announced sender as shown in Figure 6. If this happens due to packet loss, the sender will not have received an ACK replied by the receiver (ACK is attached in D-poll packet), and hence it will participate in the next reservation process to announce its intention of sending data. The discussion of the timer is given in Section 4 .

3.3.3 None, or Singular Announced Sender. If no ACK was received during reservation frame, it probably indicates that no sender has data to transmit. Hence, the receiver should immediately turn off the radio and go to the dormant state to save energy consumption. However, if only one ACK was received during the reservation frame, rather than sending a D-poll to poll the data packet, the receiver just simply keeps in the idle listening state to wait for data that will be transmitted by a sender. In the meantime, after replying an ACK to make an appointment for data polling, the sender starts a timer $T_{d p t}$ to hear D-poll at the end of the reservation frame. When the timer expired and no D-poll was received, it considers that there is no other contender in the packet polling stage, and hence it will transmit its data packet immediately even though no D-poll was received to definitely poll its data. This case is demonstrated in Figure 7. Because a data transmission could be corrupted due to collision or interference, during the waiting period, the receiver also starts another timer at the end of reservation frame to determine whether a data transmission was corrupted. The timer is set to $T_{p w t}$, which is a little larger than $T_{d p t}$. When the timer expired and no data packet was received, the receiver considers that a data collision occurs, and it sends a S-probe with a different $n$ to start an additional reservation process and differentiate the potential senders.

3.3.4 Broadcast Communications. In a receiver-initiated MAC, a broadcast transmission is regarded as a set of unicast transmissions, as illustrated by Figure 8. Specifically, if a node has a broadcast packet to transmit, it will stay in the active state for at least one sleep-wake cycle of all neighbors. The node simply disables hardware address recognition and keeps the software acknowledgment enabled. Then it acts as a regular sender by responding to each S-probe transmitted by the neighbors to which it has not sent the broadcast packet. 


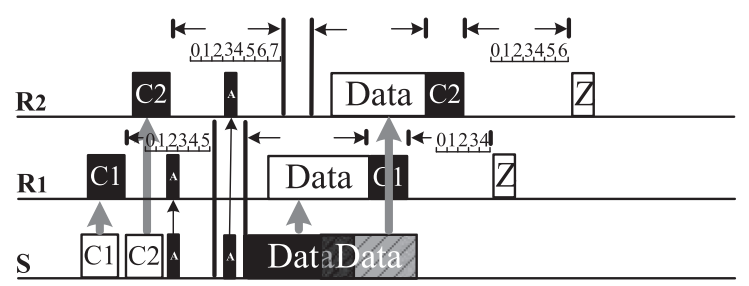

Fig. 8. Broadcast communication. By listening for different S-probes $\left(C_{1}\right.$ and $\left.C_{2}\right), S$ replies ACKs in corresponding RSVP frames, then transmits the broadcast packet just like unicast communication does.

Table 2. Parameter Values in CD-MAC

\begin{tabular}{c|c}
\hline Parameter & Value \\
\hline \hline slot duration: $T_{\text {slot }}$ & $0.4 \mathrm{~ms}$ \\
\hline first ACK delay: $T_{\text {base }}$ & $2.3 \mathrm{~ms}$ \\
\hline maximum frame size: $n_{\max }$ & 19 \\
\hline minimum frame size: $n_{\min }$ & 4 \\
\hline maximum auxiliary $m: m_{t h r}$ & 100 \\
\hline D-poll waiting timer: $T_{d p t}$ & $2 \mathrm{~ms}$ \\
\hline auxiliary data packet waiting timer: $T_{p w t}$ & $6 \mathrm{~ms}$ \\
\hline
\end{tabular}

A common case that arises with this design is the complex operations to determine whether a sender should directly transmit a broadcast packet or wait for the corresponding D-poll of each neighbor node when multiple neighbor nodes become awake during a short period of time. The unpredictable task time may cause a deviation of event time. CD-MAC addresses this problem by keeping the radio on for at least a period of time to compensate the time deviation if the previous S-probe was acknowledged. Another problem is that, while the sender is listening for the neighbors' S-probe, the sender's own S-probe timer fires. Should it send the S-probe and then return to listening or should it ignore the S-probe and continue listening? CD-MAC chooses the former.

\section{IMPLEMENTATION}

We implement CD-MAC in TinyOS [39] and run it on TelosB platform which is equipped with 802.15.4-compliant CC2420 radios. Below we discuss a few implementation-specific issues. The parameters used by CD-MAC are summarized in Table 2.

\subsection{ACK Detection}

One of the most critical issues of the proposed approach is the reservation process for senders to announce their intention of sending data to their receiver by acknowledging ACKs, and the ability of the receiver to detect these ACKs and find out who sent them, so that it can poll the right set of senders in the packet polling stage.

Note that, unlike 802.11, under 802.15.4 and in particular CC2420, an ACK contains a Data Sequence Number (DSN) but no source ID information. Therefore, the receiver cannot immediately know who the sender is just by decoding an ACK. This is where the two-pass modulo mapping method comes into play. If the receiver can accurately determine that a specific ACK was sent during the period of slot $\pi$, it can poll the sender whose ID $s$ must satisfy $\pi=\operatorname{Mod}(\operatorname{Mod}(s, m), n)$ according to Equation (1). In other words, even though the receiver may not be able to uniquely 


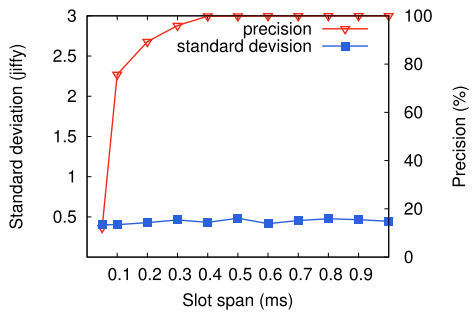

(a) Slot size

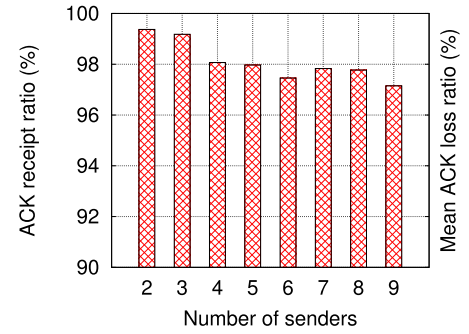

(b) ACK receipt ratio

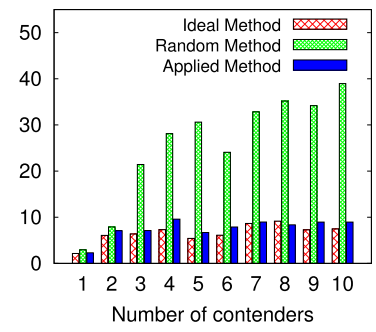

(c) ACK loss of different slot selection methods

Fig. 9. System implementation. (a) Relationship between the slot size and the prediction accuracy; (b) ACK receipt ratio when there are multiple senders; (c) ACK loss ratio of different slot selection methods.

determine $s$, the fact that the ACK is decoded correctly suggests there is a sender whose ID maps into slot $\pi$. Therefore the sender can be successfully polled to transmit its data.

To ensure sufficiently high ACK reception accuracy, the size of a reservation slot, $T_{\text {slot }}$, needs to be carefully determined based on the on-air time for transmitting an ACK, and the time variance caused by clock skew and system deviation. The former is a constant value while the latter is a variable. From the receiver's perspective, if the time of reception, say $T_{r}$, of an ACK satisfies the following condition:

$$
T_{\text {base }}+K T_{\text {slot }} \leq T_{r}<T_{\text {base }}+(K+1) T_{\text {slot }},
$$

the slot number of the received ACK is taken to be $K$. Here $T_{\text {base }}$ is the expected time gap between the completion of transmitting an S-probe and the reception of an ACK replied in the first slot. $T_{\text {base }}$ varies little and is almost constant.

An ACK is considered to have been correctly received/detected only when the detected reservation slot number calculated above matches the actual slot number a sender used. We use the probability of correct detection to measure the accuracy of our approach, and the result as a function of the slot size is shown by Figure 9(a). The results show that when the size of $T_{\text {slot }}$ is no less than $0.4 m s$ (a little longer than the time for receiving an ACK), the accuracy is higher than $99.9 \%$.

The maximum slot number, $n_{\max }$, is set to 19 according to the simulation results plotted in Figure 2. With this choice, the two-pass modulo process can reduce the ACK collision ratio to no more than $5 \%$, even if the number of potential senders can reach up to 20 . Besides, we also compute the overall ACK reception ratio when multiple senders reply ACKs during the same reservation process. This result is shown as Figure 9(b). From the figure, we can see that the ACK receipt ratio is no less than $96 \%$ when $T_{\text {slot }}$ is set to $0.4 \mathrm{~ms}$.

\subsection{Adaptive Parameter Selection}

To measure the performance of our adaptive parameter selection and slot computation, we record the amount of missed and expected ACKs of each S-probe to compute ACK loss ratio. Moreover, we evaluate two different slot computation methods which are random selection method and ideal selection method. For the former case, a sender randomly selects a slot to reply an ACK, and for the latter case, each sender replies an ACK in a fixed slot without ACK collision. The adaptive selection method combines the receiver's coordination with the senders' slot computation (simulated by the receiver). By understanding the potential neighbor nodes collected from routing packets, the receiver provides an optimal parameter pair $(n$ and $m$ ) attached in S-probe. Then, each sender computes a slot sequence number using the parameters and its node ID. Figure 9(c) shows the comparison. The ACK loss ratio of the ideal selection method (about 5\%) is due to the unreliability 
Table 3. Setting of D-poll Wait Time Threshold

\begin{tabular}{l||l|l|l|l}
\hline$T_{d p t}(\mathrm{~ms})$ & 0.5 & 1 & 2 & 3 \\
\hline \hline Positive error (\%) & 32.62 & 4.09 & 1.02 & 0.19 \\
\hline Negative error (\%) & 0.43 & 0.38 & 0.41 & 0.36 \\
\hline
\end{tabular}

of link quality. It is shown that there is no clear distinction between our adaptive selection method (called Applied Method in the figure) and the ideal selection method on ACK collision ratio with the increase of the number of senders. In addition, the ACK loss ratio of random selection method is above $20 \%$ when there is more than two senders. It may be concluded that the random selection method performs unsatisfactorily just like the random backoff in traditional MAC protocols, and the adaptive parameter selection method used by CD-MAC is near the optimal method.

\subsection{No More than One Announced Sender}

We also test the performance of the proposed method when there is no sender or a single sender responding to the receiver's transmission solicitation. As mentioned above, in this case our approach uses timers by the sender $\left(T_{d p t}\right)$ and the receiver $\left(T_{p w t}\right)$ to either trigger the data transmission or move quickly to the next round. To determine the right choice for $T_{d p t}$, we perform a set of experiments using two flows to transmit data packets independently. In any one flow, multiple senders transmit data packets to a receiver. Each sender's inter-packet interval (IPI) is set to 10 seconds, and the wake-up interval is set to $512 \mathrm{~ms}$.

In this experiment, there exist two possible wrong results: positive error and negative error. Positive error is defined as the misjudgment: when the D-poll probe is delayed, the sender infers that the receiver adopts negatively waiting and makes a wrong decision to immediately transmit data packet. Negative error means that the sender does not receive D-poll probe which has already been sent by receiver (i.e., D-poll loss). The negative error may cause packet collisions because the sender thinks there is no other contender and transmits its data packet instantly although it is not its turn to transmit the data packet. With the increase of $T_{d p t}$, the accuracy of the senders' estimation increases. We list the experimental results in Table 3. It is obvious that the negative error is stable because the D-poll loss is stable in a certain scenario. With the increase of $T_{d p t}$, the positive error decreases rapidly. To weigh the positive error and radio on time, we set $T_{d p t}$ to $2 \mathrm{~ms}$ in CD-MAC.

\section{EVALUATION}

In this section, we conduct extensive experiments to evaluate the performance of CD-MAC. Specifically, we compare the collision indicators, network reliability, and energy consumption of CDMAC with the state-of-the-art protocols, such as the widely used sender-initiated BoX-MAC [24], receiver-initiated A-MAC [8], and the recently proposed collision resolution Strawman [26]. The collision indicators include mean transmission count, mean congestion backoff count, and mean single-hop delay.

Briefly, BoX-MAC is the most typical and widely applied MAC protocol, over which the packet transmission is initiated by the sender. A-MAC is the state-of-the-art receiver-initiated link layer protocol for low-power wireless networks. A-MAC can provide the most energy efficiency compared with other receiver-initiated link layer protocols, because A-MAC adopts phase-lock mechanism for energy saving. Strawman is a contention resolution mechanism designed for receiver-initiated MACs that may experience a sudden surge of traffic and yield intense collisions. Strawman lets multiple senders contend for the next data transmission time by simultaneously 
sending contention packets with different lengths after detecting a data collision. The sender with the longest contention packet wins and then completes its transmission in the next time slot. Strawman is just activated when the link layer protocol detects data collision.

\subsection{Evaluation Setup}

For all the experiments, each node generates a packet with a fixed IPI. The packet size is set to 100 bytes. The nodes' wake-up interval and IPI are set according to experiment scenarios and will be explained in detail if needed. For each IPI, the experiment time lasts at least 30 minutes and is repeated at least five times.

We have conducted five series of experiments to evaluate the different performance of CD-MAC.

(1) First, to evaluate the main function of CD-MAC for collision avoidance, we conduct experiments by constructing one-hop networks where multiple senders transmit data packets to the same receiver;

(2) Second, we construct networks with multiple flows to evaluate the impact of inter-flow interference on the performance of MAC protocols;

(3) Third, we conduct experiments to evaluate the performance of the broadcast communication of CD-MAC;

(4) Fourth, we conduct experiments in multiple-hop networks to test the performance of the collection tree protocol (CTP [9]) building upon CD-MAC, BoX-MAC, and A-MAC, respectively;

(5) Fifth, we evaluate the tolerance of MAC protocols on hidden terminals;

(6) Finally, we test the performance of CD-MAC under the scenarios with mobility and network dynamics.

For the single-hop experiments, we allow all senders to transmit packets to their common receiver during a period of IPI for simulating burst traffic. Note that Strawman was proposed for resolving contention and it is activated once packet collision was detected by the receiver. Strawman is based on the precondition that the receiver can completely detect packet collisions. In this carefully designed experiment, because the receiver can accurately detect packet collision by counting the received packets, we can compare the performance of CD-MAC with Strawman and the other protocols experiencing burst traffic. Even though, so far, it is unreliable to accurately detect packet collisions by using the state-of-the-art wireless technique [4] in practical scenarios. Hence, except for the well-planned single-hop experiments, we won't further evaluate the performance of Strawman in the rest of experiments with practical scenarios.

In the following sections, we use packet reception ratio (PRR) as an indicator of network reliability. The energy consumption is measured by duty cycle, the portion of radio-on time, as a metric for energy efficiency. Duty cycle is a platform-independent metric. In addition, we use the single-hop time cost to denote transmission latency.

\subsection{One-Hop Collision Avoidance}

In this section, we construct single-hop networks where multiple senders transmit a data packet to an appointed receiver. The wake-up interval is set to $128 \mathrm{~ms}$, and the IPI of each sender is also set to $128 \mathrm{~ms}$ to ensure the occurrence of burst traffic load. The size of the sending queue used for buffering data packets is set to 1 , which means a new packet will be dropped if the previous packet is pending in the sending queue. The maximum retransmission count is set to the TinyOS default threshold 31.

With the same experimental configurations, such as the wake-up interval, IPI, and environmental condition, besides CD-MAC, we have also evaluated the performance of A-MAC, the A-MAC 
combined with Strawman, and BoX-MAC, respectively. For BoX-MAC with the above-mentioned configurations, when a receiver wakes up, each sender must have a packet in sending queue. By receiving a data packet, BoX-MAC extends the active state for a period of time (about $30 \mathrm{~ms}$ in TinyOS) to receive potentially upcoming packets. For A-MAC, when a receiver wakes up to poll the senders' data packets by transmitting an S-probe, each sender has a data packet in sending queue. In this case, before receiving all senders' data packets, if the receiver receives nothing after its data poll probe, the receiver can know the occurrence of data collision accurately. Hence, by combining with Strawman, A-MAC can trigger Strawman to resolve the contention in a timely manner, which is referred to as A-MAC+Strawman in the rest of this article. In our experiments, each sender records the number of retransmissions of a data packet, the backoff count of each transmission, the time cost for each data packet delivery, and the radio duty cycle, and then sends all this information to a central computer through serial ports. The receiver also records all received packets, the time cost for receiving all senders' packets, and radio duty cycle, and then sends them to the central computer in the same way.

According to the recorded information, we can compute different performance indicators of the four MAC protocols. Figure 10(a) plots the average throughput of a single-hop transmission with different number of senders. As shown in the figure, CD-MAC provides the maximum throughput compared with A-MAC, A-MAC+Strawman, and BoX-MAC. It improves the throughput by an average of $172 \%, 58.6 \%$, and $42.3 \%$, respectively. With the increase of the traffic load (i.e., the number of senders), the throughput provided by A-MAC remains steady due to the use of truncated binary exponential backoff to avoid collision. Although BoX-MAC greedily occupies the channel before each successful data delivery, it provides a higher throughput than A-MAC with the increase of traffic load. As discussed above, by using receiver-initiated MACs, senders should postpone data transmissions until the appointed receiver wakes up. Hence, when multiple senders have a data packet to forward to the same receiver, it probably leads to data collision, which is further aggravated under bursty traffic loads. By combining with Strawman to resolve this collision problem, A-MAC can improve the throughput by an average of about $60 \%$, which is close to that of BoXMAC.

We further analyze the performance of these protocols in mean transmission count, mean congestion backoff count, single-hop delay, and the duty cycle of senders. The mean transmission count is a good indicator of packet collision. However, it is hard to differentiate whether an unsuccessful transmission is due to a preamble failure or due to collision for duty-cycled sender-initiated MAC (i.e., BoX-MAC). Because BoX-MAC adopts greedy channel occupancy to transmit packet and uses congestion backoff to do channel contention, we consider both the mean transmission count (Figure 10(b)) and the congestion backoff count (Figure 10(c)) to assess the severity of data collision. As shown in Figure 10(b), the mean transmission count of A-MAC increases sharply while the mean congestion backoff count is no larger than 2 with the increase of traffic load. Packet collisions result in many retransmissions, and hence the mean single-hop delay dramatically increases, as demonstrated by Figure 10(e). By combining with Strawman, the retransmission ratio of A-MAC is sharply decreased. However, Strawman is just activated when receiver detects a collision. The starting of Strawman after a collision introduces almost $70 \%$ retransmissions. The large transmission count and low backoff count indicate that the carrier sense mechanism of traditional receiver-initiated MACs performs badly in the scenario with bursty traffic load. Unlike the other protocols, CD-MAC can effectively and efficiently differentiate potential contenders before data transmissions, and then it polls them one-by-one in the packet polling stage to fundamentally avoid collisions. The retransmission ratio of CD-MAC remains steady (about 6\%) and is consistent with the analysis of Section 4.2 . 


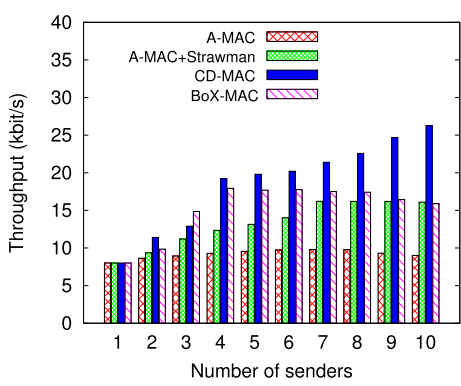

(a) Throughput

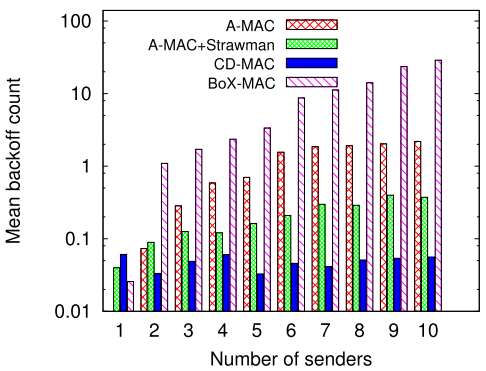

(c) Mean congestion backoff

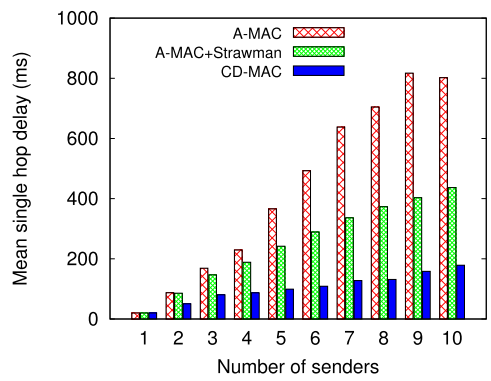

(e) Mean single hop delay

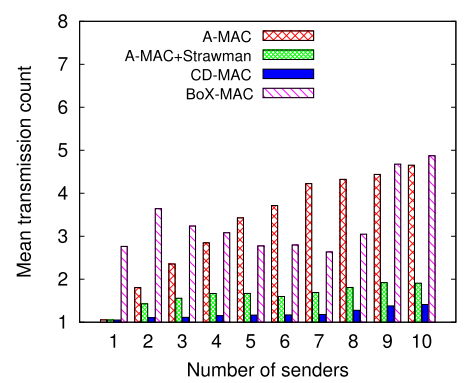

(b) Mean transmission count

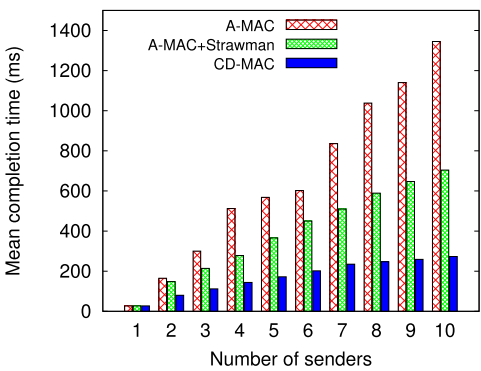

(d) Mean time cost for pulling all senders' data packets

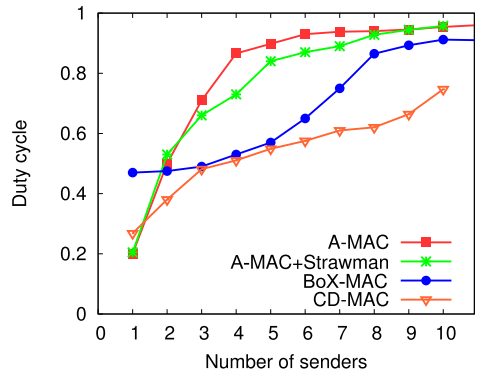

(f) Mean duty cycle of sender

Fig. 10. The performance of the single flow of BoX-MAC, A-MAC, A-MAC binding with Strawman, and CDMAC with multiple senders transmitting packets to the same next hop, the wake-up interval is set to $128 \mathrm{~ms}$ and the IPI is set to $128 \mathrm{~ms}$.

In addition, the mean completion time for a receiver to successfully receive all senders' data packets and the mean single-hop delay of each packet (from the listening of a probe to the successful delivery) are shown by Figure 10(d) and Figure 10(e), respectively. Considering the difficulty to know the equipollent single-hop delay of BoX-MAC protocols, we do not plot the related information about BoX-MAC here. As shown by the figures, the completion time of CD-MAC is far less than A-MAC and A-MAC+Strawman. As illustrated in Figure 10(f), CD-MAC is the most energy efficient protocol compared with the other three, because the high transmission count and the high packet delay cost extra energy. The change tendency of energy consumption with the variance of traffic loads matches the change tendency of the throughput and mean transmission count. CD-MAC reduces energy consumption by $23 \%$ to $39 \%$ with multiple senders compared with 


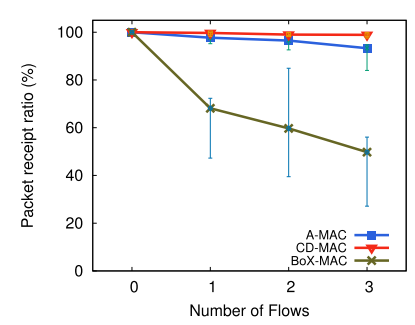

(a) PRR

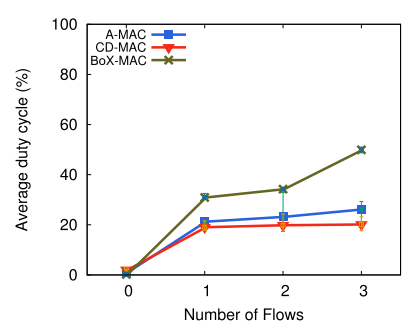

(b) Average duty cycle of senders

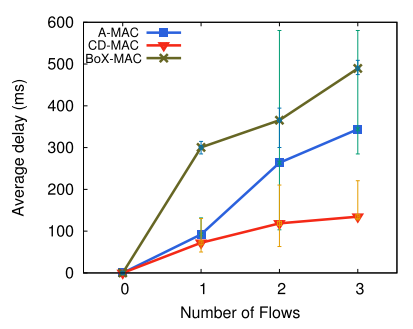

(c) Average single hop delay

Fig. 11. The performances of A-MAC, BoX-MAC, and CD-MAC in networks with multiple flows. The IPI is set to 1s. For each flow, three senders transmit packets to the same receiver. The wake-up interval is set to $512 \mathrm{~ms}$.

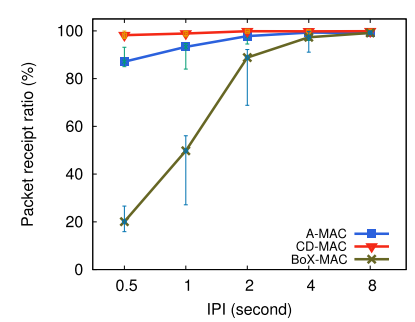

(a) PRR

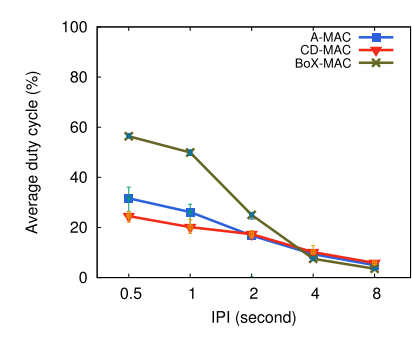

(b) Average duty cycle of senders

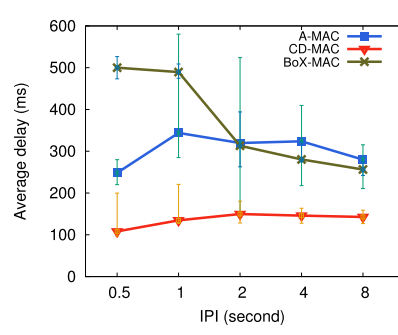

(c) Average single hop delay

Fig. 12. The performances of A-MAC, BoX-MAC, and CD-MAC in networks with only one flow. The IPI is set, respectively, to $512 \mathrm{~ms}, 1 \mathrm{~s}, 2 \mathrm{~s}, 4 \mathrm{~s}$, and $8 \mathrm{~s}$. The wake-up interval is set to $512 \mathrm{~ms}$.

A-MAC. By combining with Strawman, A-MAC slightly increases the radio duty cycle. Compared with it, CD-MAC saves energy consumption by from $18 \%$ to $30 \%$. BoX-MAC slightly decreases the duty cycle with the increase of the number of senders because multiple contenders increase the probability that the receiver is in the active state. Compared to BoX-MAC, CD-MAC saves energy consumption by $15 \%$ totally.

Overall, in the experiments for simulating bursty traffic load, CD-MAC can achieve much better performance than A-MAC, A-MAC+Strawman, and BoX-MAC, no matter the energy efficiency, network reliability, or instantaneous throughput.

\subsection{Multiple Flows Contending}

Besides the experiments with specially constructed networks with single flow explained above, we also evaluate the network performance with multiple flows in this section. In multiple-flow networks, all nodes in the network are within the transmission range of each other. To change the traffic load, we can change the number of independent flows and the IPI of each sender. Each flow is constructed as three senders and a receiver, and the senders transmit data packets to the same receiver. For each experiment, each sender generates data packets with a fixed IPI. We test each clique network with five different IPIs which are 512ms, 1s, 2s, 4s, and 8s, respectively. All nodes' wake-up interval is set to $512 \mathrm{~ms}$. By carefully scheduling all senders' data generation plan for each clique network, all senders of a specific flow can generate data packets within one wake-up interval. The evaluation results are illustrated in Figures 11 and 12, where 0 flow on the horizontal axis of Figures 11(a), 11(b), and 11(c) indicates there is no traffic load in the network, and thus all energy consumption is consumed by the periodic wake-up phase. By changing the number of flows and changing the IPI to test the performance of $\mathrm{CD}-\mathrm{MAC}, \mathrm{A}-\mathrm{MAC}$, and BoX-MAC, we respectively 
analyze the packet delivery performance, energy efficiency of senders, and transmission delay. Figures 11(a), 11(b), and 11(c) illustrate the corresponding performance with different number of flows, and Figures 12(a), 12(b), and 12(c) plot the performance by setting different IPIs.

5.3.1 Packet Receipt Ratio. Figure 11(a) and Figure 12(a) illustrate the change of PRR with the increasing number of contending flows and the increase of IPIs of each sender, respectively. As shown by them, the PRR of CD-MAC is always close to $100 \%$. BoX-MAC can deliver most of the generated data packets when there are no more than 1 flow; however, the PRR drops quickly when the number of flows reaches 2 or 3 . As shown in the figure, when there are three flows in a network, CD-MAC can improve PRR by about 55\% compared to BoX-MAC. Because there is less channel occupancy for data transmission, the PRR of A-MAC is less influenced by the changing of the number of flows than that of BoX-MAC. However, with the increase of traffic load, A-MAC shows significant performance degradation because of the increasing packet collision and longdelayed retransmission scheme. By using phase-lock mechanism, when a transmission fails or a sender misses its receiver's wake-up phase, the sender using A-MAC has to wait for receiver's next wake-up time for retransmission. Compared with A-MAC's 85\% PRR, the PRR of CD-MAC can be up to $99.3 \%$ when IPI is set to $512 \mathrm{~ms}$. The high packet-delivery performance of CD-MAC benefits from the proposed contention resolution in this article which can instantaneously differentiate all potential senders after a receiver's wake-up and then poll them one-by-one in a conflict-free manner. Hence, in CD-MAC, packet collisions are far less than that of A-MAC.

5.3.2 Duty Cycle of Senders. As shown in Figure 11(b) and Figure 12(b), A-MAC can achieve extreme low-power consumption when traffic load is low. However, with the increase of traffic load or the number of network flows, both A-MAC and BoX-MAC gradually perform worse than CDMAC. As illustrated in Figure 11(b), with the increase of the number of traffic flows, CD-MAC can respectively save more energy than A-MAC by about $7 \%$, and more than BoX-MAC by about $25 \%$, because CD-MAC can quickly finish data polling for potential multiple senders compared with A-MAC and BoX-MAC. With the decrease of traffic load in Figure 12(b), the advantage of CDMAC over A-MAC and BoX-MAC decreases gradually. When the IPI is set to 4 seconds, the duty cycle of CD-MAC is slightly higher than A-MAC and BoX-MAC due to the accumulation of more energy consumption during each reservation frame. Even so, when IPI is less than 4 seconds, CDMAC significantly outperforms them in energy efficiency. The main reason is that the additional energy consumption for differentiating potential senders during reservation phase is far less than the energy consumption for resolving potential data collision and channel contention during data polling/receiving phase.

5.3.3 Single-Hop Delay. Figure 11(c) and Figure 12(c) plot the mean single-hop delay with different traffic flows and traffic loads. The single-hop delay of CD-MAC increases slowly with the increasing number of flows, and almost remains steady with the increasing traffic load. Unlike CD-MAC, the mean delay of A-AMC and BoX-MAC increases sharply with the increase of flow number. The changing tendency of BoX-MAC's mean delay is caused by the contention of all nodes in the network, and the high delay of A-MAC is also caused by the interference from other flows, because the total channel occupancy time of A-MAC in the scenario with the three flows are longer than CD-MAC so that the probability of packet collision increases.

Overall, CD-MAC outperforms A-MAC and BoX-MAC in terms of packet reception ratio, energy efficiency, and transmission delay in network scenarios with multiple flows. The performance improvement of CD-MAC benefits from the quick data polling with very low collision ratio and the effective contention resolution mechanism. 
Table 4. Broadcast Packet Delivery Ratio under Different Network Conditions

\begin{tabular}{c|c|c|c}
\hline $\begin{array}{c}\text { Different network } \\
\text { conditions }\end{array}$ & \multicolumn{3}{|c}{ Broadcast packet delivery ratio } \\
mean & min & max \\
\hline \hline Static network & $99.79 \%$ & $97.54 \%$ & $100 \%$ \\
\hline Dynamic network & $96.85 \%$ & $85.93 \%$ & $100 \%$ \\
\hline
\end{tabular}

\subsection{Performance of Broadcast Communication}

To evaluate the performance of broadcast communication of CD-MAC, we conduct experiments in indoor testbed with 30 Telosb nodes under different network conditions: static network with low data traffic (IPI is set to 1 minute) and dynamic network with high data traffic (IPI is set to 20 seconds). The experiment is divided into two steps: The first is the determination of neighbors for each network node; The second step is to determine whether a broadcast packet can be delivered to all the neighbors. Due to the lack of standard neighbor determination metric, we run CTP to determine the neighbors of each node. By collecting the neighbor node set of each node, all network nodes take turns to transmit broadcast packet. Each node transmits 100 broadcast packets, and each broadcast communication lasts for one wake-up interval. All network nodes overhear potential broadcast packets and feed back the receiving information to central computer through wire-cable. We calculate the broadcast packet delivery ratio (PDR) and show the results in Table 4 . In a static network, broadcast communication is reliable enough to deliver a broadcast packet to almost all neighbors. On the other hand, in dynamic network situation, high data traffic affects the performance of broadcast communication because multiple concurrent senders may result in slot collision and data collision. Although the broadcast packet delivery ratio under the dynamic network is relatively lower than that of the static network, the averaged delivery performance $(96.85 \%)$ is good enough to support for upper-layer flooding protocols.

\subsection{Collection Tree Protocol Performance}

In this section, we explore how well the Collection Tree Protocol (CTP) [9] performs over CDMAC. CTP is the default data collection protocol in TinyOS [39] and has been widely applied in practical application. It represents the link layer client by delivering/extracting packets to/from the underlying MAC protocol. In an indoor testbed with 30 Telosb nodes as shown by Figure 13, by setting the output power of RF to level 2 (less than $-25 \mathrm{dBm}$ ), the testbed nodes form a multi-hop network. The sink node is placed at the bottom left corner of the testbed. The default TinyOS MAC implementation (BoX-MAC), A-MAC, ContikiMAC [7], and CD-MAC are tested, respectively, in the same testbed with the same configurations. Note that by using BoX-MAC and ContikiMAC, the radio chip of the sink node is set to always-on. To evaluate the impact of the sudden surge of traffic load on the performance of testbed network, we set two different traffic patterns for these MACs to respectively simulate the absence or presence of bursty traffic. For each traffic pattern, we evaluate each MAC protocol by repeating each pattern several times and having each pattern last for at least 5 hours:

Periodic Traffic (PT): Each node generates a data packet every 1 minute. In this traffic pattern, we can evaluate the energy efficiency of each MAC protocol under the scenarios with low traffic load.

Bursty Traffic (BT): We also construct burst traffic load by selecting 10 neighboring senders in testbed network to continuously produce data packets lasting for 5 minutes. In this case, the IPI of the selected 10 nodes is set to $1 \mathrm{~s}$. When a new data packet is generated and the previous data 


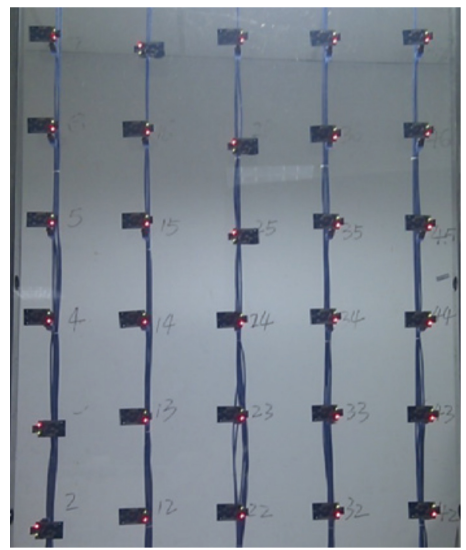

Fig. 13. Topology of indoor testbed. Thirty TelosB nodes are selected for data collection, sink node is placed in the left bottom corner of the testbed.

Table 5. CTP Performance over BoX-MAC, A-MAC, and CD-MAC with Periodic Traffic

\begin{tabular}{c|c|c|c|c}
\hline PT & BoX-MAC & A-MAC & CD-MAC & ContikiMAC \\
\hline \hline Duty Cycle & $5.14 \%$ & $3.35 \%$ & $3.53 \%$ & $2.79 \%$ \\
\hline Average PDR & $99.3 \%$ & $99.1 \%$ & $99.7 \%$ & $99.99 \%$ \\
\hline
\end{tabular}

Table 6. CTP Performance over BoX-MAC, A-MAC, and CD-MAC with Bursty Traffic

\begin{tabular}{c|c|c|c|c}
\hline BT & BoX-MAC & A-MAC & CD-MAC & ContikiMAC \\
\hline \hline Duty Cycle & $7.15 \%$ & $5.58 \%$ & $4.14 \%$ & $6.72 \%$ \\
\hline Average PDR & $95.8 \%$ & $97.8 \%$ & $99.3 \%$ & $47.9 \%$ \\
\hline
\end{tabular}

packet hasn't been successfully delivered, the new packet is put into the sending buffer. This traffic pattern can evaluate the reliability of CTP protocol built upon different MAC protocols which resolve channel contention and data collision under the scenarios with sudden surge of traffic.

In this experiment, besides the data-packet forwarding, each node also records the duty cycle for its radio chip and reports it to sink node by attaching to the data packets generated by itself. Then, we analyze the performance of CTP by using CD-MAC, BoX-MAC, A-MAC, and Contiki$\mathrm{MAC}$, respectively. The network-wide data delivery ratio and radio duty cycle are listed in Table 5 and Table 6. The results show that CD-MAC provides steady and high performance under different traffic loads. Although the energy consumption of CD-MAC is slightly more than that of A-MAC in very low periodic traffic due to the indispensable reservation stage, it provides more energy efficiency in the scenario with bursty traffic. Furthermore, CD-MAC provides the highest network reliability than BoX-MAC and A-MAC. In addition, compared with CD-MAC, ContikeMAC is much more energy efficient and works well in low data traffic networks. However, in the burst traffic network, the failure of the phase-lock mechanism and the use of fast sleeping make the performance of ContikiMAC significantly lower than that of CD-MAC.

Besides, the additional control overhead of CD-MAC can be measured approximately in networks with extremely low traffic load, by which the reservation process cannot play a practical 


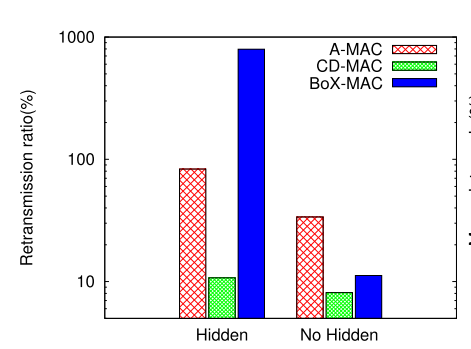

(a) Packet retransmission ratio

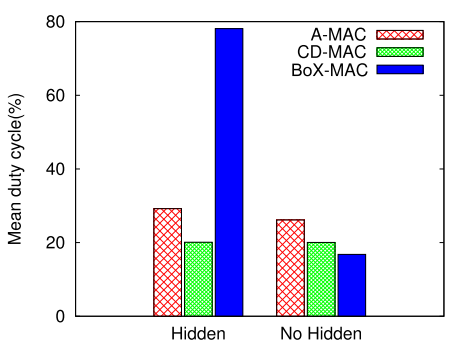

(b) Radio duty cycle of sender

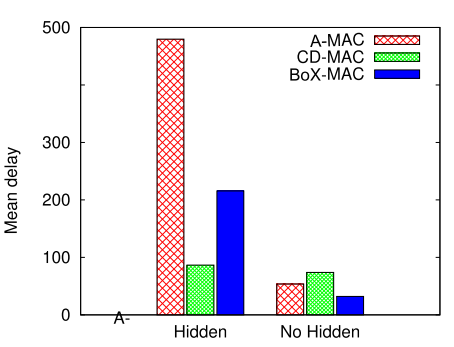

(c) Single hop delay

Fig. 14. Performance of CD-MAC, A-MAC, and BoX-MAC with and without hidden terminals. (a) The mean ratio of the retransmission count to the number of total received packets, (b) the gap of the mean duty cycle between the hidden terminal scenario and the no hidden terminal scenario, and (c) the mean delay.

role other than to consume extra energy for detecting potential senders. In these experimental scenarios with periodic traffic, by setting each node's IPI to 1 minute, we can evaluate (approximately) the impact of the control overhead of CD-MAC on energy efficiency under low traffic load. As given in Table 5, the radio duty cycle of CD-MAC (3.53\%) is slightly higher than A-MAC (3.35\%).

\subsection{Network with Hidden Terminal}

We also run BoX-MAC, A-MAC, and CD-MAC in several controlled scenarios to determine their ability to handle hidden terminals. In this experiment, we construct two different network topologies: one with hidden nodes and the other with no hidden nodes. Specifically, for each case, we set up a network with three nodes where two senders transmit data packets to the same receiver. Both the wake-up interval and IPI are set to $512 \mathrm{~ms}$. For BoX-MAC, the radio of all nodes is set to always on. The distance between each sender and the receiver is the same. In the case with no hidden terminal, the two senders are also within each other's transmission range, whereas in the case with hidden terminal, the two senders are not aware of each other.

The experiment results are plotted in Figure 14. We compare the retransmission ratio of BoXMAC, A-MAC, and CD-MAC in Figure 14(a). As shown in the figure, the $x$-axis indicates whether the two senders are hidden from each other (denoted as Hidden) or not (denoted as No Hidden). To guarantee a perfect link, we control the transmission power under which the packet receipt ratio between a sender and a receiver is almost $100 \%$. With the case of hidden terminal, BoXMAC experiences a high transmission failure ratio (795\% retransmission ratio) due to collision, because the senders are not aware of each other and each node uses deterministic backoff time causing successive retransmission collisions. However, in the scenario with no hidden terminal, the retransmission ratio (11\%) is far less than that of hidden terminal scenario. In addition, hidden terminals also greatly degrade system performance in terms of energy consumption. The duty cycle increases from $16.8 \%$ to $78 \%$, as shown in Figure 14(b), and the mean latency increases from $32 \mathrm{~ms}$ to $215 \mathrm{~ms}$, as illustrated by Figure 14(c). For A-MAC, although it uses truncated exponential backoff to avoid collision, it has little effect on collision avoidance because it is activated after the occurrence of a data collision. As shown in Figure 14(a), although the difference of retransmission ratios between the hidden terminal scenario (83.2\%) and the no hidden terminal scenario (33.7\%) is not as large as BoX-MAC, it increases the energy consumption by about 3\%, as shown by Figure 14 (b). Surprisingly, the mean delay increases from $54 \mathrm{~ms}$ to $479 \mathrm{~ms}$, as shown in Figure 14 (c). The mismatch between the duty cycle and the mean delay of A-MAC is because A-MAC adopts a phase-lock mechanism to save energy, i.e., during the waiting period, senders turn off the radio until the next time the receiver wakes up. 


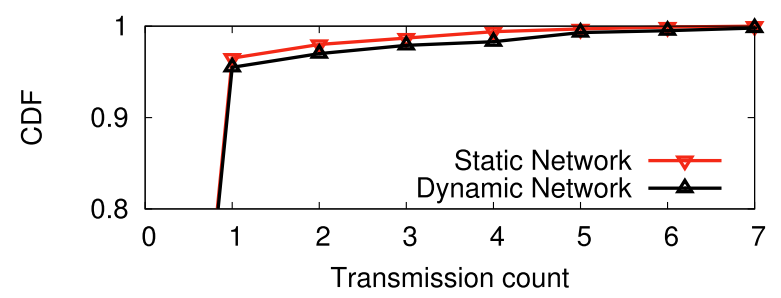

Fig. 15. The CDF of single-hop transmission count in static network and dynamic network.

According to the experimental results, we can conclude that CD-MAC outperforms BoX-MAC and A-MAC for resolving hidden terminal problem. It is resilient against hidden terminals because it can differentiate the potential hidden nodes using different slot number during the reservation phase. As demonstrated by the experimental results, the difference of the retransmission ratio between the hidden terminal scenario $(10.7 \%)$ and the no hidden terminal scenario $(8.2 \%)$ is insignificant for CD-MAC compared with other protocols. Under this condition, the extra energy consumption for the hidden terminal is only $0.5 \%$, and the increase in delay is about $13 \mathrm{~ms}$.

\subsection{Adaptation to Network Dynamics}

5.7.1 Topology Change. To test the adaptability of CD-MAC to node arrival and departure in dynamic networks, we regularly reset some selected nodes (departure and arrival of node) during the period of network experiment with periodic traffic as mentioned above. This experiment is conducted in the indoor testbed with 30 Telosb nodes. The sink node is placed in a corner of the testbed, and all nodes' radio power level is set to level 2 to form multi-hop network. Each network node periodically generates data packet or receives data packets from child nodes, and then forwards them to the next hop parent node. As sender, each node records the number of transmission count of each data packet. As a resetting node, it records the time taken for joining the existing network. And as the neighbor or parent of the resetting nodes, network nodes record the change of parameter set $n$ and $m$ due to the arrival or departure of potential senders.

Note that a transmission failure is denoted as receiving an S-probe from next hop node but no acknowledgment for the pending data packet. According to the recorded transmission information, we plot the CDF of the single-hop transmission count in Figure 15. As shown by the figure, compared with the scenario without node resetting, CD-MAC is robust enough to tolerate network dynamics, and there is no clear performance difference between the two experiments. Actually, the flexible reservation scheme according to the parent's parameters contributes to the capability of adapting to mobility and dynamics.

In addition, the resetting of network nodes could trigger a route request event and could pull route information from its neighbor nodes. Note that each network node periodically wakes up and transmits data poll probe to notify its neighbors/child nodes. Hence, the new arrival could quickly determine its route. According to our evaluation results, the vast majority of new arrival nodes could find the optimal route within the period of one wake-up interval.

We also analyze the effect of network dynamics on the recalculation of parameters $n$ and $m$. Note that the departure of network nodes cannot increase (actually can decrease) the expected slot collision ratio and actual collision ratio. Hence, the previous parameter can also effectively differentiate potential senders. We calculate the proportion of the changes of $n$ and $m$ when a different number of new arrivals becomes the child nodes of a given network node, and plot the results in Table 7. As it turns out, if the number of new arrivals is no more than 3, only a small fraction of network nodes (less than 13\%) are triggered to recalculate the values of $n$ and $m$, 
Table 7. The Proportion of Triggering Recalculation of the Optimal Parameters $n$ and $m$ When Different Number of New Arrivals Become the Children of a Given Network Node

\begin{tabular}{l||l|l|l|l|l}
\hline Number of new arrivals & 1 & 2 & 3 & 4 & 5 \\
\hline \hline Proportion of recalculation & $1.6 \%$ & $4.3 \%$ & $12.9 \%$ & $25.6 \%$ & $37.8 \%$ \\
\hline
\end{tabular}

Table 8. Impact of External Interference on Performance

\begin{tabular}{|c||cc|cc|cc|cc|}
\hline \multirow{2}{*}{$\begin{array}{c}\text { Network } \\
\text { scenarios }\end{array}$} & \multicolumn{2}{|c|}{ PDR } & \multicolumn{2}{c|}{ Duty cycle } & \multicolumn{2}{c|}{ Single hop delay (ms) } & \multicolumn{2}{c|}{ Retransmission ratio } \\
\hline \hline WiFi interference & $99.31 \%$ & $91.05 \%$ & $4.37 \%$ & $6.38 \%$ & 91 & 221 & $9.2 \%$ & $173.5 \%$ \\
No interference & $99.42 \%$ & $97.79 \%$ & $4.09 \%$ & $5.60 \%$ & 83 & 168 & $7.9 \%$ & $87.4 \%$ \\
\hline
\end{tabular}

otherwise, the joining of new arrival does not affect the effectiveness of previous parameters. The robustness benefits from the mechanism of collision ratio threshold introduced in Section 3.2.3. However, under the scenarios with high dynamics, network nodes should frequently recalculate the optimal parameter to guarantee low collision ratio. As shown in the table, when more than five new arrivals simultaneously become the child nodes of a given node, about $37.8 \%$ of the network nodes are triggered to recalculate the optimal parameter.

5.7.2 External Interference. To evaluate the impact of external interference on the performance of CD-MAC, we also conduct experiments in the indoor testbed with two different scenarios: (1) Network nodes are interfered with by WiFi by using the 19th channel of ZigBee which is overlapped with WiFi frequency spectrum; and (2) free of interference from WiFi by using the 26th channel. The network traffic pattern in this experiment is the same as the bursty traffic introduced in Section 5.5: In the testbed network, we randomly select 10 neighboring senders in testbed network to continuously produce data packets that last 5 minutes. We respectively analyze the PDR, radio duty cycle, averaged single-hop delay, and averaged single-hop transmission count of CDMAC under the two network scenarios, and give the experimental results in Table 8. As shown in the table, the impact of external interference on the performance of CD-MAC is significantly smaller than on the performance of A-MAC. The robustness and reliability of CD-MAC mainly benefits from the mechanism of utilizing temporal diversity of quick responses of all potential senders to differentiate them and poll their data packets in a conflict-free manner, and the mechanism for soliciting the senders whose ACK/data was previously corrupted again after polling the data packets of all detected senders.

Interference affects the performance of CD-MAC in two ways: ACK collision and data packet collision. For the first case, if ACKs of potential senders are all corrupted at the receiver caused by interference, the receiver would think that there is no sender and then it quickly turns off the radio to save energy, which could increase the transmission delay and energy consumption at the senders. For this severe case in duty-cycled networks, no better way could be used to make a perfect tradeoff between energy efficiency and transmission reliability under the premise that the receiver can only capture the channel situation. By far, the current receiver-initiated MACs still cannot solve this problem well. However, compared with the traditional receiver-initiated MACs, the utilization of temporal diversity of multiple quickly replied ACKs could significantly increase the chance of ACK collision tolerance. Take Figure 1 as an example. The loss of $S_{5}$ 's ACK in the first reservation process has little effect on the overall transmission delay because the receiver will start 
an additional reservation process for potential senders after polling $S_{3}$ 's data packet. For the other case, the loss of a data packet (e.g., $S_{4}$ 's data packet) doesn't mean the sender should retransmit the data packet in the receiver's next wake-up phase. After the first round of the packet polling stage, the sender has another opportunity to send the corrupted data packet again. By enhancing the tolerance of $\mathrm{ACK}$ and data packet collision, $\mathrm{CD}-\mathrm{MAC}$ could provide more reliable and robust data forwarding performance compared to the state-of-the-art receiver-initiated MACs.

\section{DISCUSSIONS}

In this section, we further discuss some important issues of CD-MAC.

Additional Overhead: Compared to the traditional receiver-initiated MAC, such as A-MAC, the possibly additional overhead of CD-MAC is only the reservation process for utilizing the temporal diversity of quickly replied ACKs from potential senders in the scenarios with very low traffic load. The additional overhead of CD-MAC depends on the practical network scenarios, such as networks with a sudden surge of traffic or stable traffic, or networks with extremely low network traffic load or high network traffic load. In network scenarios with extremely low traffic load, the feature of collision-catching in receiver-initiated MAC is not serious, hence CD-MAC can't take full advantage of its collision avoidance mechanism by utilizing the temporal diversity of quickly replied ACKs from potential senders other than consuming extra energy for detecting potential senders. With the decreasing of traffic load, compared with the state-of-the-art receive-initiated MACs, the performance improvement of CD-MAC is also decreasing. Even so, CD-MAC also has almost comparable level of performance compared with the state-of-the-art receiver-initiated MAC.

High Dynamics and Mobility: CD-MAC is not applicable to a network with high dynamics and mobility. The frequent arrival or departure of nodes in these networks could result in significant energy consumption for recomputing the optimal parameters $n$ and $m$, and the double-module process may lose effect when network topology has been significantly changed.

Applicable to Different Network Scenarios: The generality of CD-MAC is characterized by the feature that it is applicable to different network scenarios, such as periodic data collection scenario, event-driven or query-driven data collection scenarios, and the like, because the proposed contention avoidance mechanism in this article can commendably resolve the innate collisioncatching problem of receiver-initiated MACs. On that basis, CD-MAC can quickly forward the received/generated data packets; hence, it can guarantee energy efficiency and data delivery reliability in network scenarios with any high traffic load or low traffic load. In practical sensor networks, the majority of low traffic loads is usually accompanied by an unexpected surge of high traffic. Hence, the design of CD-MAC for solving collision-catching problem in receiver-initiated MACs is necessary and meaningful to significantly extend its application scenarios.

\section{CONCLUSION}

In this article, we present CD-MAC, an adaptive receiver-initiated link layer that offers low duty cycle in light traffic loads, achieves contention avoidance in bursty traffic loads, and significantly diminishes the influence of the hidden terminal problem. In addition, it is adaptive to network dynamics, such as topology change and node mobility.

We implemented CD-MAC in TinyOS and evaluated its performance on an indoor Testbed with TelosB nodes. In scenarios with bursty traffic loads, evaluation results show that CD-MAC performs better than the stat-of-the-art MAC protocols. In addition, the experimental results also demonstrate that CD-MAC can effectively mitigate the influence of hidden terminal problems.

This article has been previously published in IEEE SECON 2015. Compared with the conference version, in this article, we have added the following content: 
(1) Introduction of the principle of broadcast and the evaluation of the performance of broadcast.

(2) Discussion on the impact of inter-flow on performance of CD-MAC.

(3) Comparison the performance of CD-MAC with ContikiMAC.

(4) Evaluation of the effect of network dynamics on the robustness of CD-MAC.

Based on the contention avoidance mechanism, it is interesting to combine CD-MAC with other upper-layer protocol to further improve network performance. We leave it to future work.

\section{ACKNOWLEDGMENTS}

We sincerely thank the editor and anonymous reviewers for insightful comments to improve our work. We are extremely grateful for the constant help of Dr. Xiaopei Wu.

\section{REFERENCES}

[1] L. Sherly Puspha Annabel and K. Murugan. 2015. Energy-efficient quorum-based MAC protocol for wireless sensor networks. ETRI fournal 15, 7 (2015), 5074-5117. DOI : https://doi.org/10.4218/etrij.15.0114.0688

[2] A. Arora, P. Dutta, S. Bapat, V. Kulathumani, H. Zhang, and V. Naik. 2004. A line in the sand: A wireless sensor network for target detection, classification, and tracking. Computer Networks 46, 5 (2004), 605-634. DOI : https://doi. org/10.1016/j.comnet.2004.06.007

[3] Michael Buettner, Gary V. Yee, Eric Anderson, and Richard Han. 2006. X-MAC: A short preamble MAC protocol for duty-cycled wireless sensor network. In Proceedings of the 4th International Conference on Embedded Networked Sensor Systems (SenSys'06). ACM, Boulder, Colorado, 307-320.

[4] Gregory Chockler, Murat Demirbas, Seth Gilbert, Calvin Newport, and Tina Nolte. 2005. Consensus and collision detectors in wireless ad hoc networks. In Proceedings of the 24th Annual ACM Symposium on Principles of Distributed Computing (PODC'05). ACM, New York, 197-206. DOI : https://doi.org/10.1145/1073814.1073850

[5] Domenico De Guglielmo, Giuseppe Anastasi, and Marco Conti. 2013. A localized slot allocation algorithm for wireless sensor networks. In Proceedings of Ad Hoc Networking Workshop (MED-HOC-NET'13). IEEE, ACM, Ajaccio, 89-96. DOI : https://doi.org/10.1109/MedHocNet.2013.6767415

[6] Ilker Demirkol, Cem Ersoy, and Fatih Alagoz. 2006. MAC protocols for wireless sensor networks: A survey. IEEE Communications Magazine 44, 4 (May 2006), 115-121. DOI : https://doi.org/10.1109/MCOM.2006.1632658

[7] Adam Dunkels. 2011. The ContikiMAC Radio Duty Cycling Protocol. SICS Technical Report T2011:13 (2011).

[8] Prabal Dutta, Stephen Dawson-Haggerty, Yin Chen, Chieh-Jan Mike Liang, and Andreas Terzis. 2012. A-MAC: A versatile and efficient receiver-initiated link layer for low-power wireless. ACM Transactions on Sensor Networks 8, 4 (2012), 30:1-30:29. DOI : https://doi.org/10.1145/2240116.2240119

[9] Omprakash Gnawali, Rodrigo Fonseca, Kyle Jamieson, David Moss, and Philip Levis. 2009. Collection tree protocol. In Proceedings of the 7th International Conference on Embedded Networked Sensor Systems (SenSys'09). ACM, California, 1-14. DOI : https://doi.org/10.1145/1644038.1644040

[10] D. J. Goodman, R. Valenzuela, K. T. Gayliard, and B. Ramamurthi. 1989. Packet reservation multiple access for local wireless communications. IEEE Transactions on Communications 37, 8 (Aug. 1989), 885-890. DOI: https://doi.org/10. $1109 / 26.31190$

[11] Vehbi Gungor and Gerhard Hancke. 2009. Industrial wireless sensor networks: Challenges, design principles, and technical approaches. IEEE Transactions on Industrial Electronics 56, 10 (2009), 4258-4265. DOI : https://doi.org/10.1109/ TIE.2009.2015754

[12] Harvey Motulsky and Ransnas Lennart.1987. Fitting curves to data using nonlinear regression: A practical and nonmathematical review. The FASEB fournal 1, 5 (Nov. 1987), 365-374.

[13] Tian He, Sudha Krishnamurthy, John A. Stankovic, Tarek Abdelzaher, Liqian Luo, Radu Stoleru, Ting Yan, Lin Gu, Jonathan Hui, and Bruce Krogh. 2004. Energy-efficient surveillance system using wireless sensor networks. In Proceedings of the 2nd International Conference on Mobile Systems, Applications, and Services (MobiSys'04). ACM, 270-283. DOI : https://doi.org/10.1145/990064.990096

[14] Jonathan. W. Hui and David Culler. 2004. The dynamic behavior of a data dissemination protocol for network programming at scale. In Proceedings of the 2nd International Conference on Embedded Networked Sensor Systems (SenSys'04). ACM, 81-94. DOI : https://doi.org/10.1145/1031495.1031506

[15] Rhee Injong, Warrier Ajit, Aia Mahesh, Min Jeongki, and Mihail L. Sichitiu. 2008. Z-MAC: A hybrid MAC for wireless sensor networks. IEEE/ACM Transactions on Networking 16, 3 (2008), 511-524. DOI : https://doi.org/10.1109/TNET. 2007.900704 
[16] Texas Instruments. 2006. Cc2420: $2.4 \mathrm{ghz}$ ieee 802.15.4/zigbee-ready rf transceiver. http://www.chipcon.com/files/ CC2420_Data_Sheet_1_3.pdf.

[17] Kyle Jamieson, Hari Balakrishnan, and Y. C. Tay. 2006. Sift: A MAC protocol for event-driven wireless sensor networks. In Proceedings of the Third European Conference on Wireless Sensor Networks (EWSN'06). Springer-Verlag, Zurich, Switzerland, 260-275. DOI: https://doi.org/10.1007/11669463_2

[18] Xiaoyu Ji, Yuan He, Jiliang Wang, Wei Dong, Xiaopei We, and Yunhao Liu. 2014. Walking down the STAIRS: Efficient collision resolution for wireless sensor networks. In Proceedings of the 33rd Annual IEEE International Conference on Computer Communications (INFOCOM'14). IEEE, Toronto, ON, 961-969.

[19] Philip Levis, Neil Patel, David Culler, and Scott Shenker. 2004. Trickle: A self-regulating algorithm for code propagation and maintenance in wireless sensor networks. In Proceedings of the 1st Conference on Symposium on Networked Systems Design and Implementation (NSDI'04). USENIX Association, San Francisco, California, 2-2.

[20] Daibo Liu, Zhichao Cao, Jiliang Wang, Yuan He, Mengshu Hou, and Yunhao Liu. 2016. Duplicate detectable opportunistic forwarding in duty cycled wireless sensor networks. IEEE/ACM Transactions on Networking 24, 2 (2016), 662-673. DOI : https://doi.org/10.1109/TNET.2014.2387440

[21] Yunhao Liu, Yuan He, Mo Li, Jiliang Wang, Kebin Liu, and Xiangyang Li. 2013. Does wireless sensor network scale? A measurement study on greenorbs. IEEE Transactions on Parallel and Distributed Systems 24, 10 (2013), 1983-1993. DOI : https://doi.org/10.1109/TPDS.2012.216

[22] Xufei Mao, Xin Miao, Yuan He, Xiangyang Li, and Yunhao Liu. 2012. CitySee: Urban CO2 monitoring with sensors. In Proceedings of the 31rd Annual IEEE International Conference on Computer Communications (INFOCOM'12). IEEE, Orlando, FL, 1611-1619. DOI : https://doi.org/10.1109/INFCOM.2012.6195530

[23] Lufeng Mo, Yuan He, Yunhao Liu, Jizhong Zhao, Shao-Jie Tang, Xiang-Yang Li, and Guojun Dai. 2009. Canopy closure estimates with GreenOrbs: Sustainable sensing in the forest. In Proceedings of the 7th ACM Conference on Embedded Networked Sensor Systems (SenSys'09). ACM, New York, 99-112. DOI : https://doi.org/10.1145/1644038.1644049

[24] David Moss and Philip Levis. 2008. BoX-MACs: Exploiting Physical and Link Layer Boundaries in LowPower Networking. Technical Report SING-08-00, Stanford University (2008).

[25] Razvan Musaloiu-E., Chieh-Jan Mike Liang, and Andreas Terzis. 2008. Koala: Ultra-low power data retrieval in wireless sensor networks. In Proceedings of the 7th International Conference on Information Processing in Sensor Networks (IPSN'08). ACM, Washington, DC, 421-432. DOI : https://doi.org/10.1109/IPSN.2008.10

[26] Fredrik Osterlind, Luca Mottola, Thiemo Voigt, Nicolas Tsiftes, and Adam Dunkels. 2012. Strawman: Resolving collisions in bursty low-power wireless networks. In Proceedings of the 11th International Conference on Information Processing in Sensor Networks (IPSN'12). ACM, Beijing, China, 161-172. DOI : https://doi.org/10.1109/IPSN.2012.6920954

[27] Maria Rita Palattella, Nicola Accettura, and Xavier Vilajosana. 2012. Standardized protocol stack for the internet of (important) things. IEEE Communications Surveys \& Tutorials 15, 3 (2012), 1389-1406. DOI : https://doi.org/10.1109/ SURV.2012.111412.00158

[28] Pangun Park, Carlo Fischione, A. Bonivento, Karl H. Johansson, and Alberto Sangiovanni-Vincent. 2011. Breath: An adaptive protocol for industrial control applications using wireless sensor networks. IEEE Transactions on Mobile Computing 10, 6 (2011), 821-838. DOI : https://doi.org/10.1109/TMC.2010.223

[29] Joseph Polastre, Jason Hill, and David Culler. 2004. TDM-based coordinationfunction (TCF) in WLAN for high throughput. In Proceedings of the Global Telecommunications Conference (GLOBECOM'04). IEEE, Dallas, Texas, 32353239. DOI : https://doi.org/10.1109/GLOCOM.2004.1378948

[30] Joseph Polastre, Jason Hill, and David Culler. 2004. Versatile low power media access for wireless sensor networks. In Proceedings of the 2nd International Conference on Embedded Networked Sensor Systems (SenSys'04). ACM, Baltimore, MD, 95-107. DOI : https://doi.org/10.1145/1031495.1031508

[31] Joseph Polastre, Jason Hill, and David Culler. 2004. Versatile low power media access for wireless sensor networks. In Proceedings of the 2Nd International Conference on Embedded Networked Sensor Systems (SenSys'04). ACM, Baltimore, MD, 95-107. DOI : https://doi.org/10.1145/1031495.1031508

[32] Abdul Razaque and Khaled M. Elleithy. 2014. Energy-efficient boarder node medium access control protocol for wireless sensor networks. Sensors 14, 3 (2014), 5074-5117. DOI : https://doi.org/10.3390/s140305074

[33] Kannan Srinivasan, Maria A. Kazandjieva, Saatvik Agarwal, and Philip Levis. 2008. The \&\#946;-factor: Measuring wireless link burstiness. In Proceedings of the 6th ACM Conference on Embedded Network Sensor Systems (SenSys'08). ACM, Raleigh, NC, 29-42.

[34] Yanjun Sun, Shu Du, Omer Gurewitz, and David B. Johnson. 2008. DW-MAC: A low latency, energy efficient demandwakeup MAC protocol for wireless sensor networks. In Proceedings of the 9th ACM International Symposium on Mobile Ad Hoc Networking and Computing (MobiHoc'08). ACM, New York, NY, USA, 53-62. DOI : https://doi.org/10.1145/ 1374618.1374627

[35] Yanjun Sun, Omer Gurewitz, and David B. Johnson. 2008. RI-MAC: A receiver-initiated asynchronous duty cycle MAC protocol for dynamic traffic loads in wireless sensor networks. In Proceedings of the 6th International Conference 
on Embedded Networked Sensor Systems (SenSys'08). ACM, Raleigh, NC, 1-14. DOI : https://doi.org/10.1145/1460412. 1460414

[36] Lei Tang, Yanjun Sun, Omer Gurewitz, and David B. Johnson. 2011. EM-MAC: A dynamic multichannel energyefficient MAC protocol for wireless sensor networks. In Proceedings of the 12th ACM International Symposium on Mobile Ad Hoc Networking and Computing (MobiHoc'11). ACM, Article 23, 11 pages. DOI: https://doi.org/10.1145/ 2107502.2107533

[37] Lei Tang, Yanjun Sun, Omer Gurewitz, and David B. Johnson. 2011. PW-MAC: An energy-efficient predictive-wakeup MAC protocol for wireless sensor networks. In INFOCOM. IEEE, 1305-1313. http://dblp.uni-trier.de/db/conf/infocom/ infocom2011.html\#TangSGJ11.

[38] Y. C. Tay, Kyle Jamieson, and Hari Balakrishnan. 2006. Collision-minimizing CSMA and its applications to wireless sensor networks. IEEE Journal on Selected Areas in Communications 22, 6 (Sept. 2006), 1048-1057. DOI : https://doi.org/ 10.1109/JSAC.2004.830898

[39] TinyOS-2.1.1. 2010. http://www.tinyos.net/.

[40] Xiaopei Wu, Mingyan Liu, and Yue Wu. 2012. In-situ soil moisture sensing: Optimal sensor placement and field estimation. ACM Transactions on Sensor Networks 8, 4 (Sept. 2012), 33:1-33:30. DOI : https://doi.org/10.1145/2240116. 2240122

[41] Y. Z. Zhao, M. Ma, C. Y. Miao, and T. N. Nguyen. 2010. An energy-efficient and low-latency MAC protocol with adaptive scheduling for multi-hop wireless sensor networks. Comput. Commun. 33, 12 (July 2010), 1452-1461. DOI : https://doi.org/10.1016/j.comcom.2010.03.025

Received August 2017; revised September 2018; accepted March 2019 